\title{
Exploring the Specificity of Rationally Designed Peptides Reconstituted from the Cell-Free Extract of Deinococcus radiodurans toward $\mathrm{Mn}(\mathrm{II})$ and $\mathrm{Cu}(\mathrm{II})$
}

Peana Massimiliano, ${ }^{*}, \dagger$ Elzbieta Gumienna-Kontecka, ${ }^{*}+{ }^{\dagger}$ Francesca Piras,${ }^{\dagger}$ Malgorzata Ostrowska, ${ }^{\dagger}$ Karolina Piasta, ${ }^{\ddagger}$ Karolina Krzywoszynska, ${ }^{\circledR}$ Serenella Medici, ${ }^{\dagger}$ and Maria Antonietta Zoroddu ${ }^{\dagger}$

†Department of Chemistry and Pharmacy, University of Sassari, Via Vienna 2, 07100 Sassari, Italy.

${ }^{\ddagger}$ Faculty of Chemistry, University of Wrocław, 14 F. Joliot-Curie St. 50-383 Wrocław, Poland.

${ }^{\S}$ Public Higher Medical Professional School in Opole, Katowicka 68, 45-060 Opole, Poland.

* Corresponding Authors

Massimiliano Peana - Department of Chemistry and Pharmacy, University of Sassari, 07100 Sassari, Italy;

(1) orcid.org/0000-0002-3306-0419; Email: peana@uniss.it

Elzbieta Gumienna-Kontecka - Faculty of Chemistry, University of Wrocław, 50-383 Wrocław, Poland;

orcid.org/0000-0002-9556-6378; Email: elzbieta.gumienna-kontecka@chem.uni.wroc.pl

Figures and Tables 
DEHGTAVMLK (DP1)

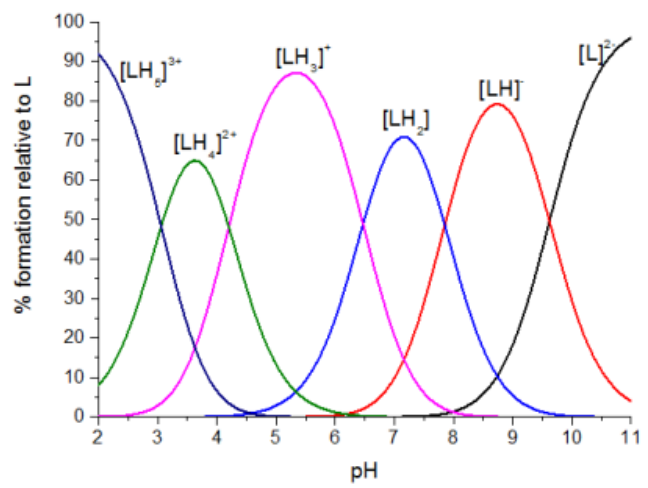

GTAVMLKDEH (Term-DEH)

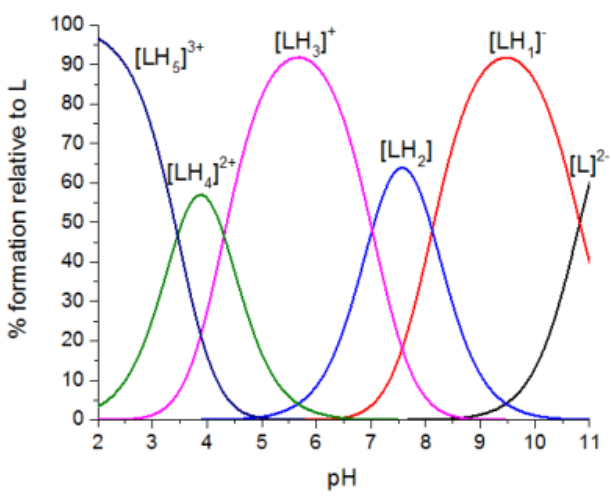

DEHGGGGDEH (Bis-DEH)

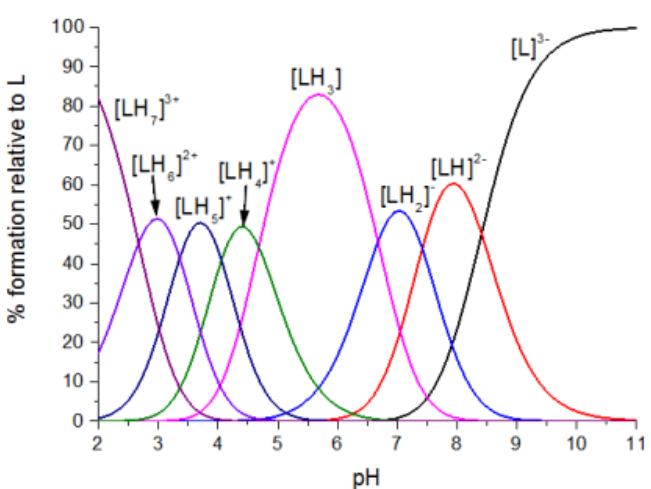

THMVLAKGED (DP2)

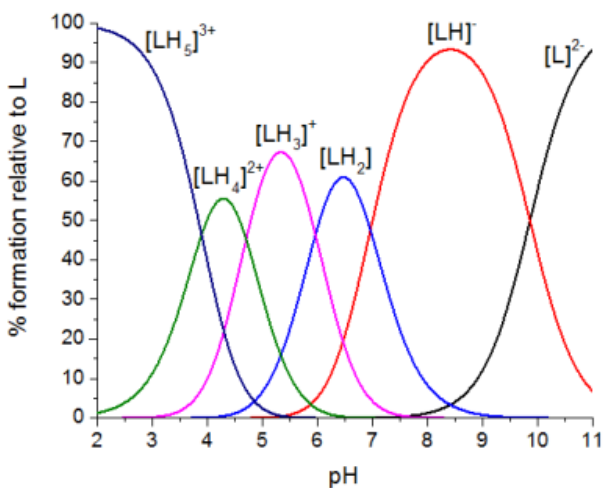

TMVLDEHAKG (Mid-DEH)

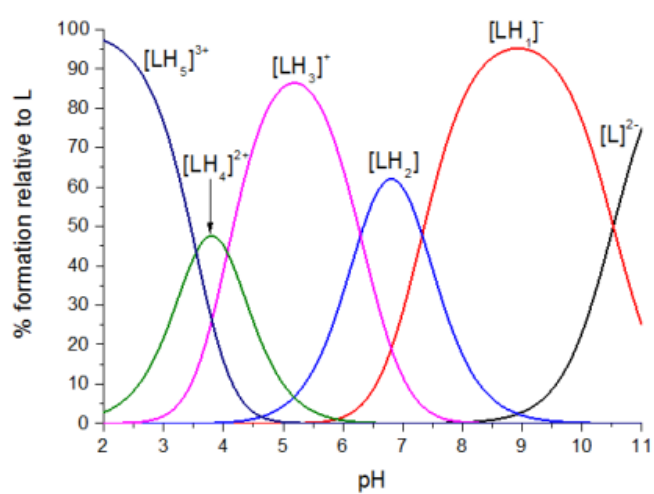

Figure S1. Representative distribution diagram for the protonation equilibria of DEHGTAVMLK (DP1), THMVLAKGED (DP2), GTAVMLKDEH (Term-DEH), TMVLDEHAKG (Mid-DEH) and DEHGGGGDEH (Bis$\mathrm{DEH}$ ), at $\mathrm{T}=298 \mathrm{~K}$ and $I=0.1 \mathrm{M} \mathrm{NaClO}_{4}$. 
a

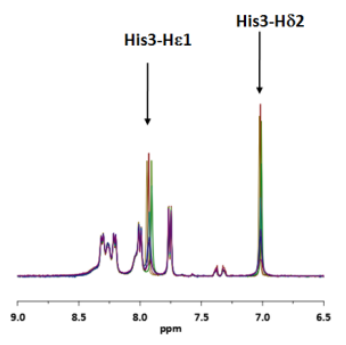

C

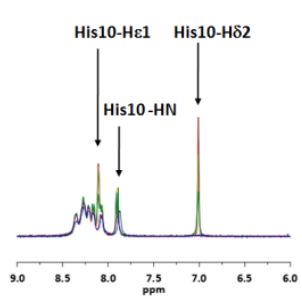

Cu(II):GTAVMLKDEH (Term-DEH)

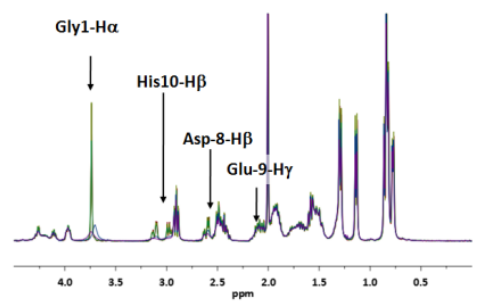

e

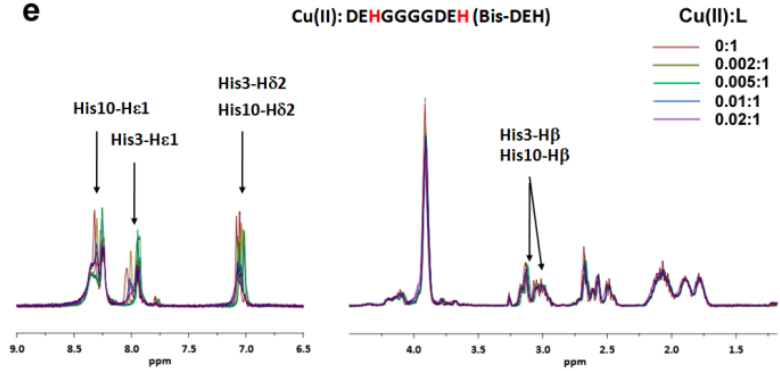

b

Cu(II): THMVLAKGED (DP2)
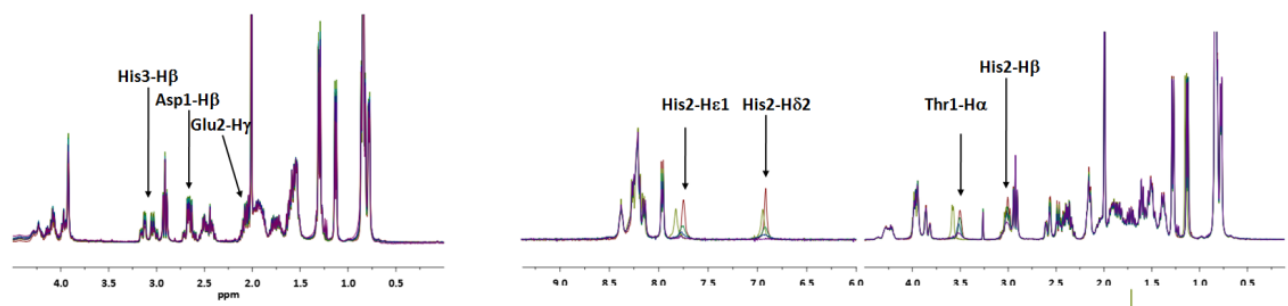

d

Cu(II):TMVLDEHAKG (Mid-DEH)

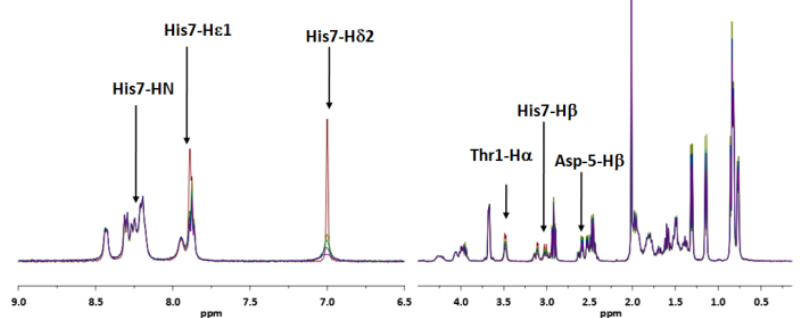

Figure S2. Superposition of 1D ${ }^{1} \mathrm{H}$ NMR spectra for a) DEHGTAVMLK (DP1), b) THMVLAKGED (DP2), c) GTAVMLKDEH (Term-DEH), d) TMVLDEHAKG (Mid-DEH) and e) DEHGGGGDEH (Bis-DEH) peptides by increasing amounts of $\mathrm{Cu}(\mathrm{II})$ from 0:1 to $0.02: 1$ metal to ligand molar ratio, at $\mathrm{pH} \sim 7$. The residues experiencing the largest relaxation effects are labeled in the spectra and highlighted along the sequence in red color. 

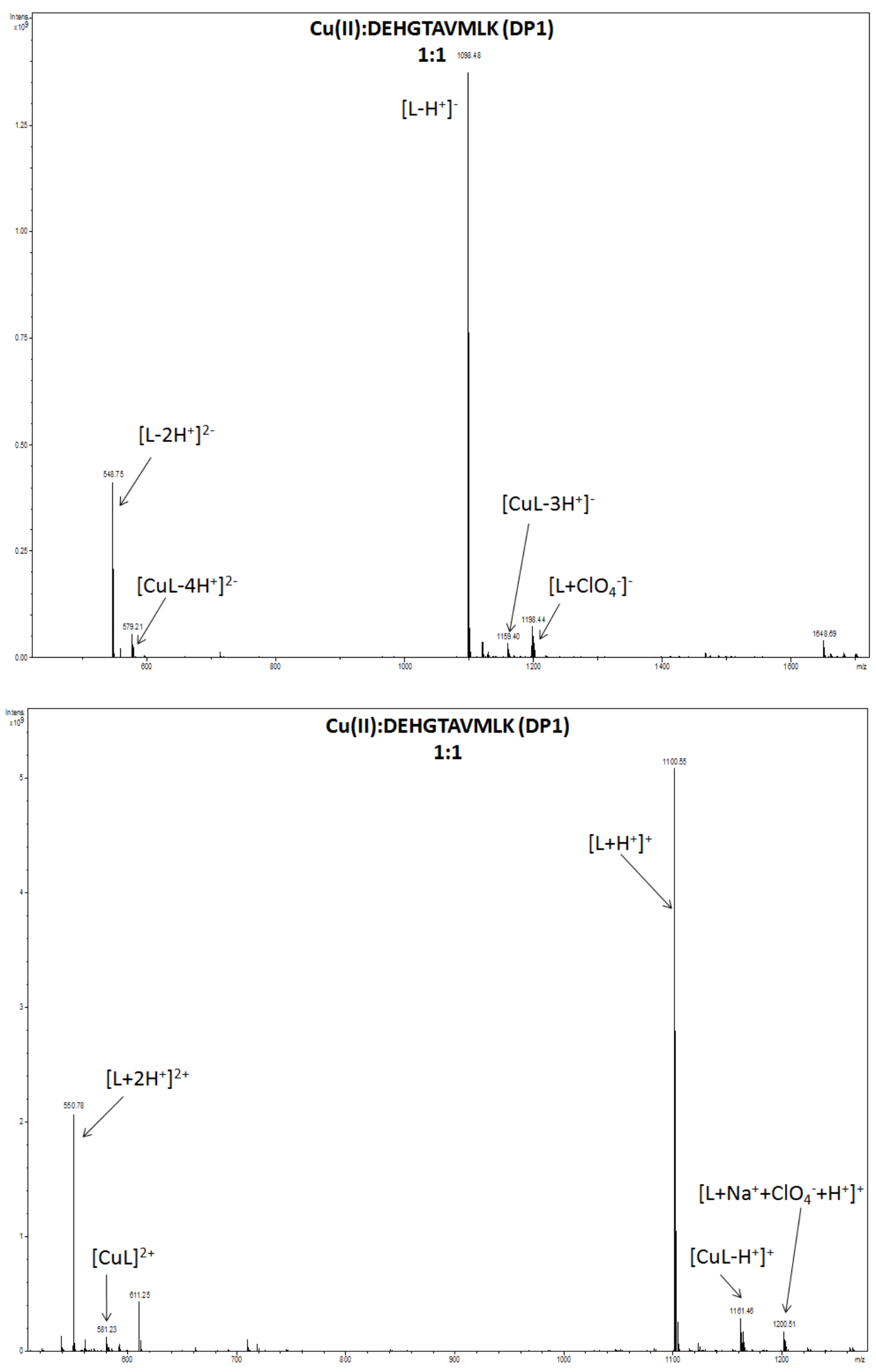

Figure S3. ESI-MS of $\mathrm{Cu}(\mathrm{II})$ :DEHGTAVMLK (DP1), at 1:1 molar ratio and pH 7. 


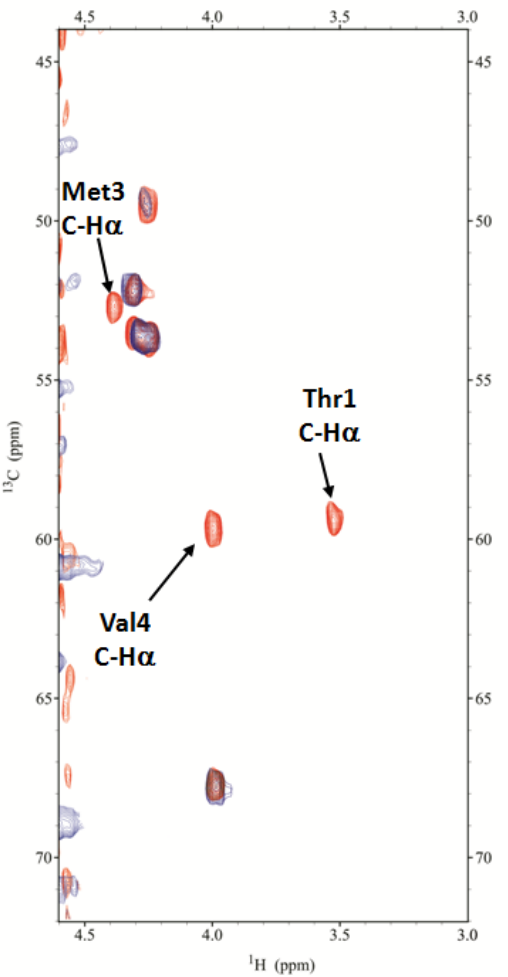

Figure S4. Aliphatic region of ${ }^{1} \mathrm{H}-{ }^{13} \mathrm{C}$ HSQC spectrum of DP2 free (red) and $\mathrm{Cu}(\mathrm{II}): \mathrm{DP} 2$ system at 0.02:1 molar ratio (blue), and pH 10. Disappearing peaks are labeled. 

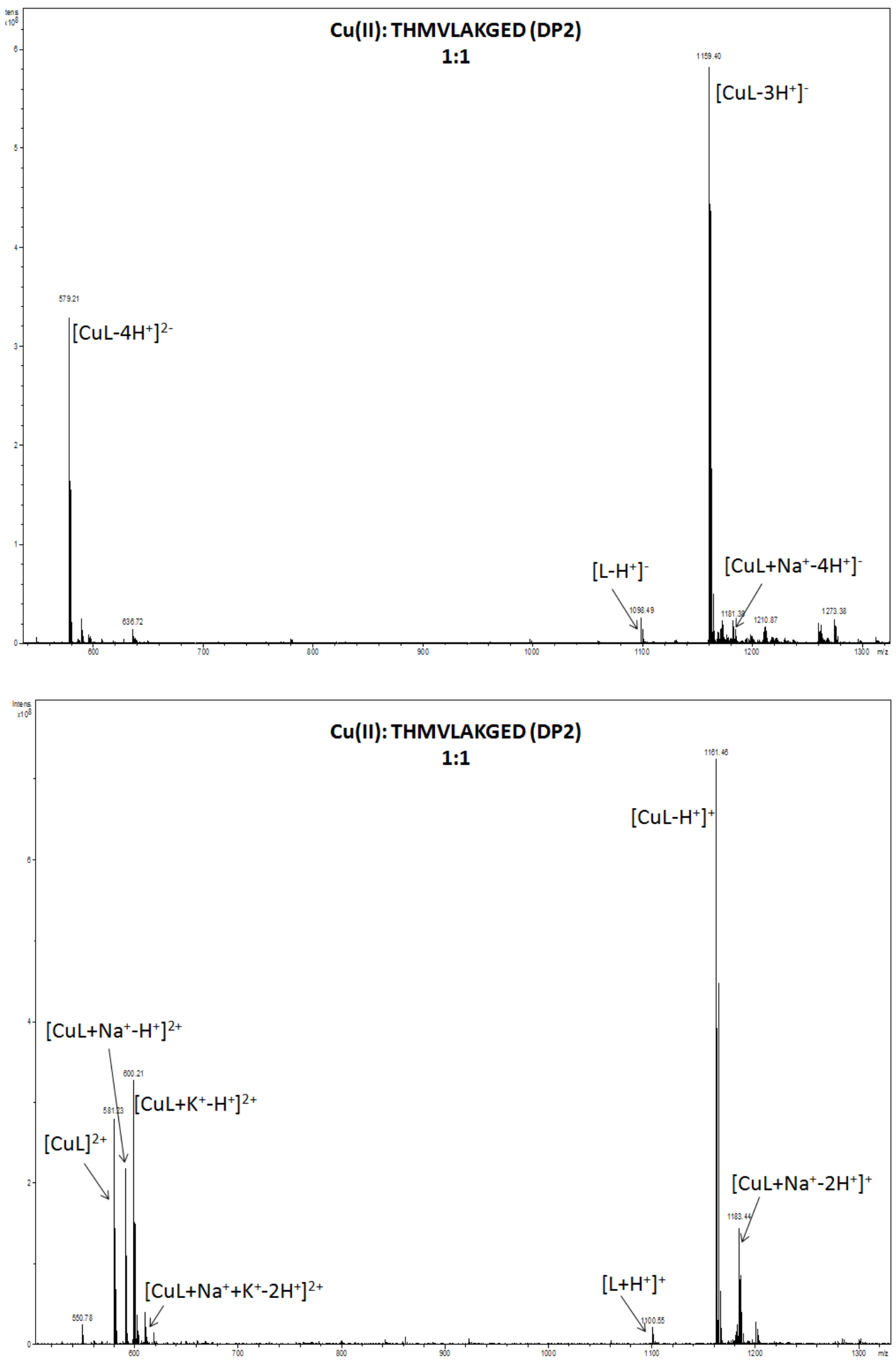

Figure S5. ESI-MS of $\mathrm{Cu}(\mathrm{II})$ :THMVLAKGED (DP2), at 1:1 molar ratio, and pH 7. 


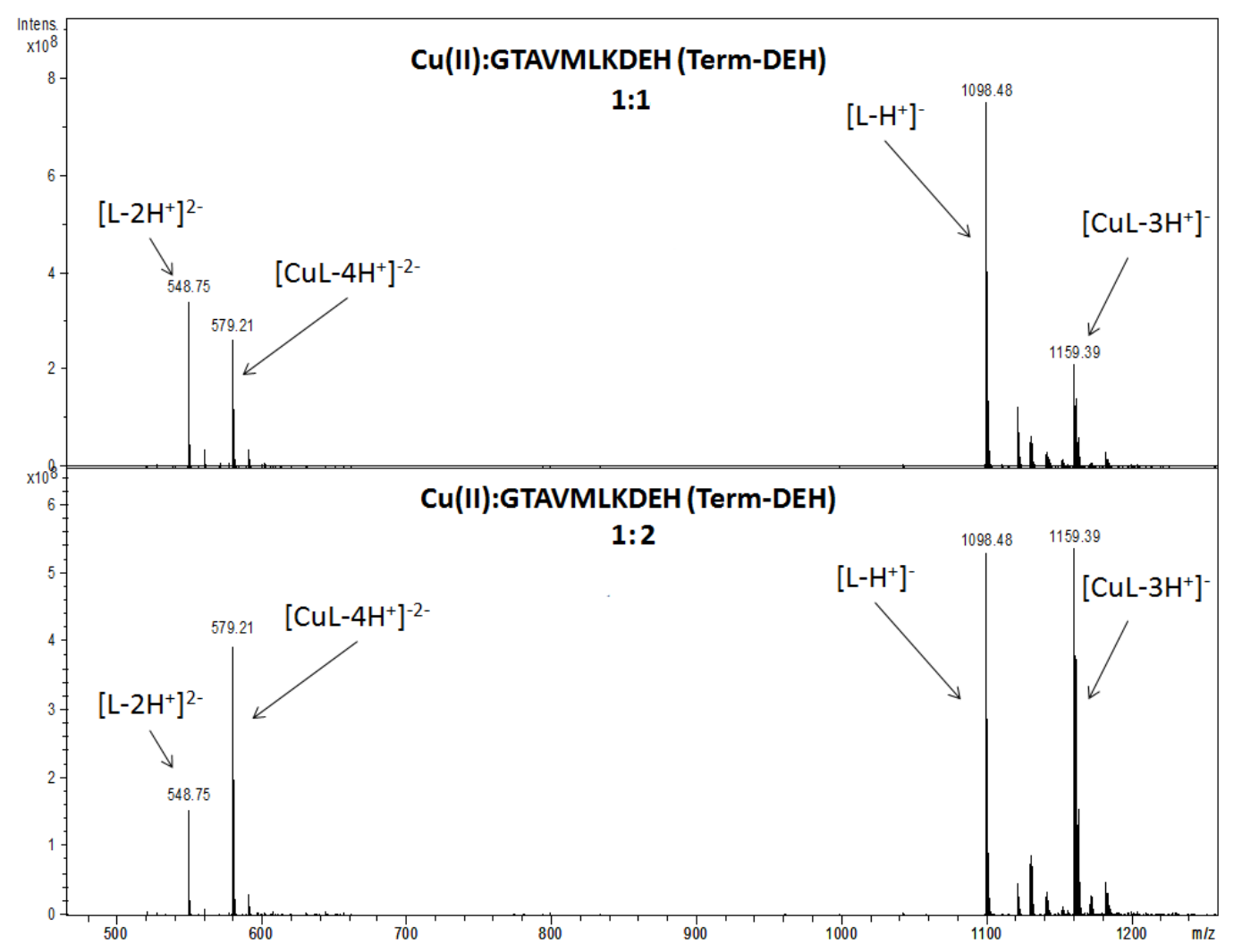

Figure S6. ESI-MS of Cu(II):GTAVMLKDEH (Term-DEH), at 1:1 and 1:2 molar ratio, and pH 7. 


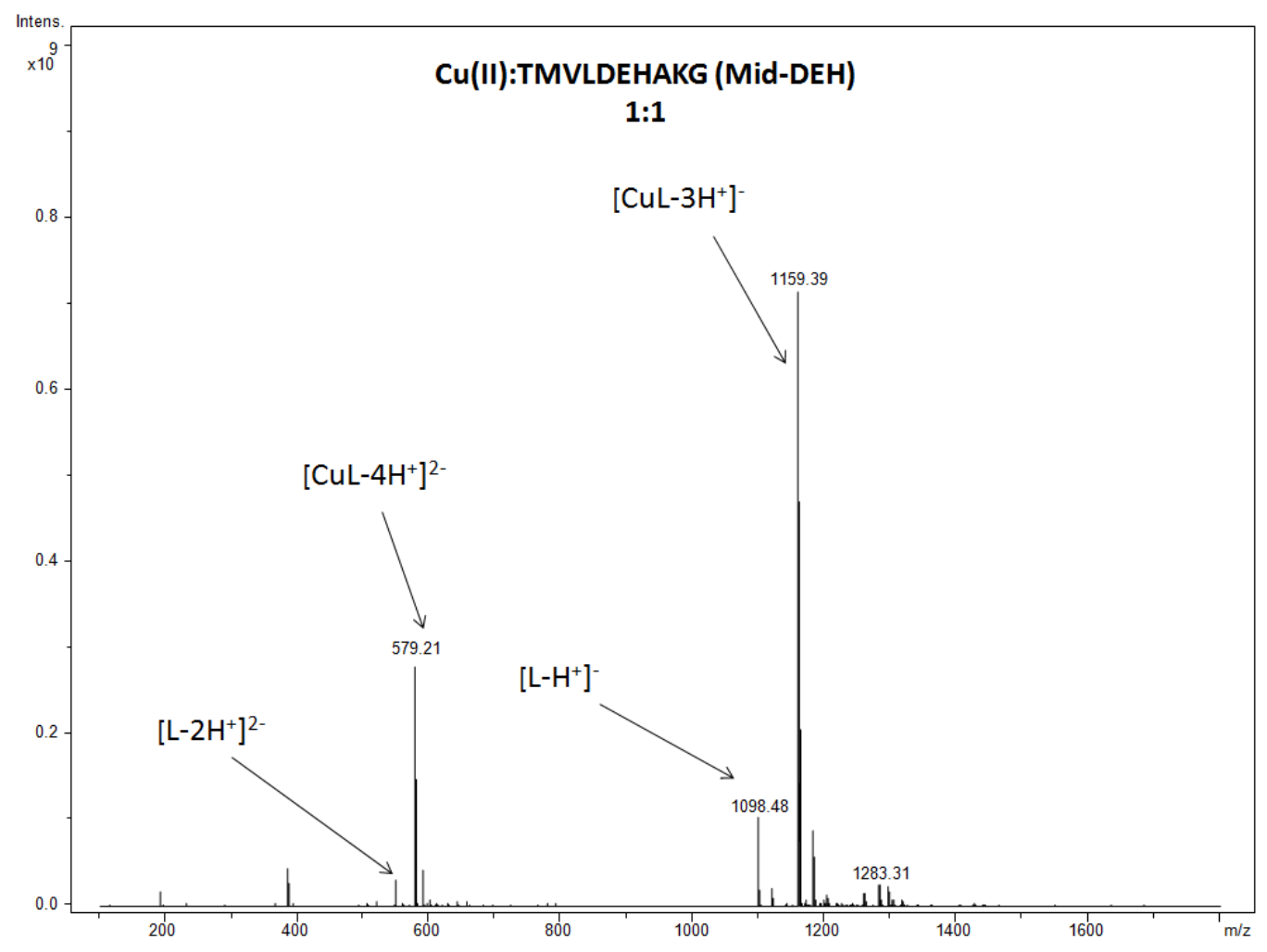

Figure S7. ESI-MS of Cu(II):TMVLDEHAKG (Mid-DEH), at 1:1 molar ratio, and pH 7. 


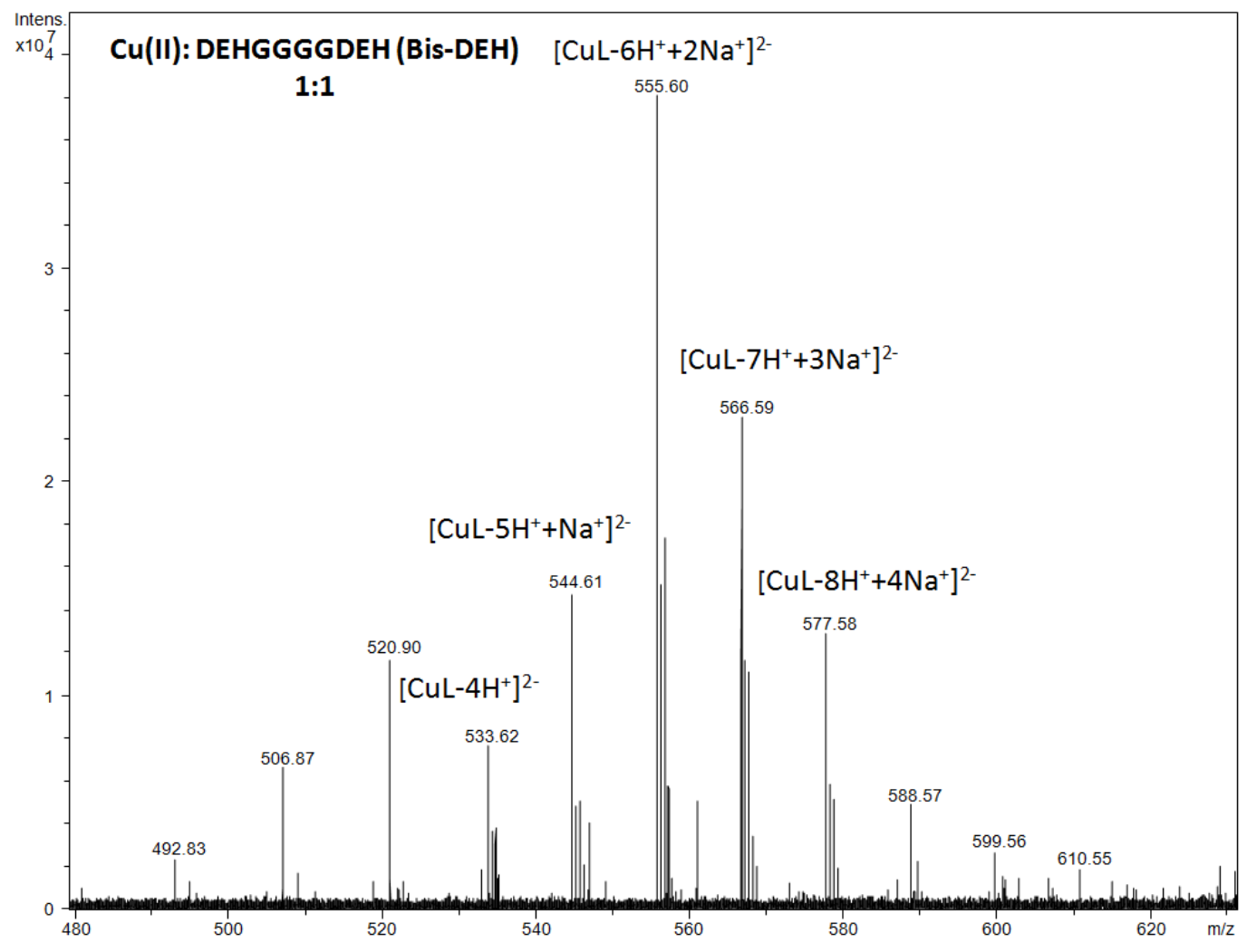

Figure S8. ESI-MS of $\mathrm{Cu}(\mathrm{II})$ :DEHGGGGDEH (Bis-DEH), at 1:1 molar ratio, and $\mathrm{pH} 7$. 

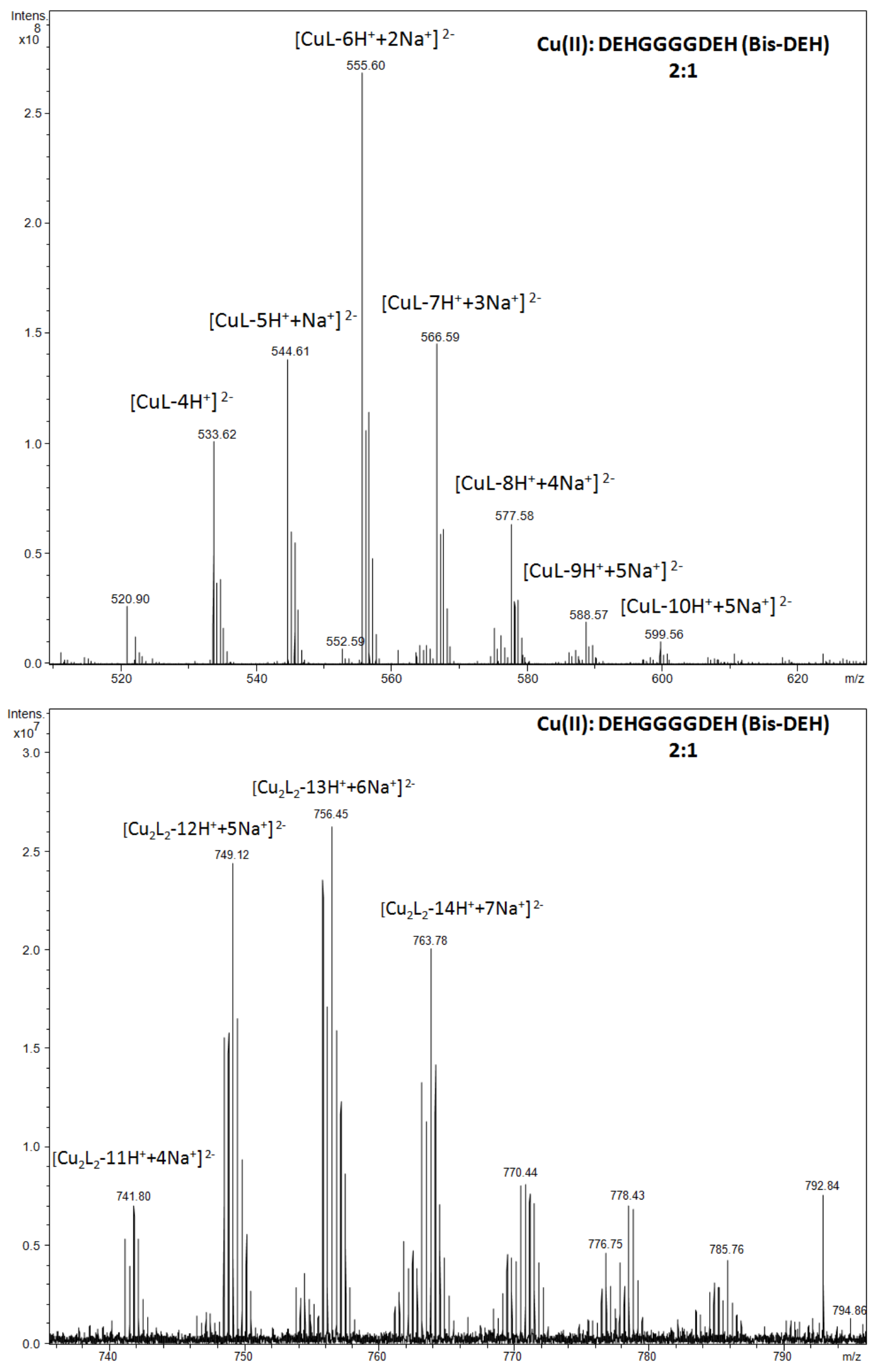

Figure S9. ESI-MS of $\mathrm{Cu}(\mathrm{II})$ :DEHGGGGDEH (Bis-DEH) at 2:1 molar ratio, and pH 7. 


\section{Mn(II):GTAVMLKDEH (Term-DEH)}

1.1:1
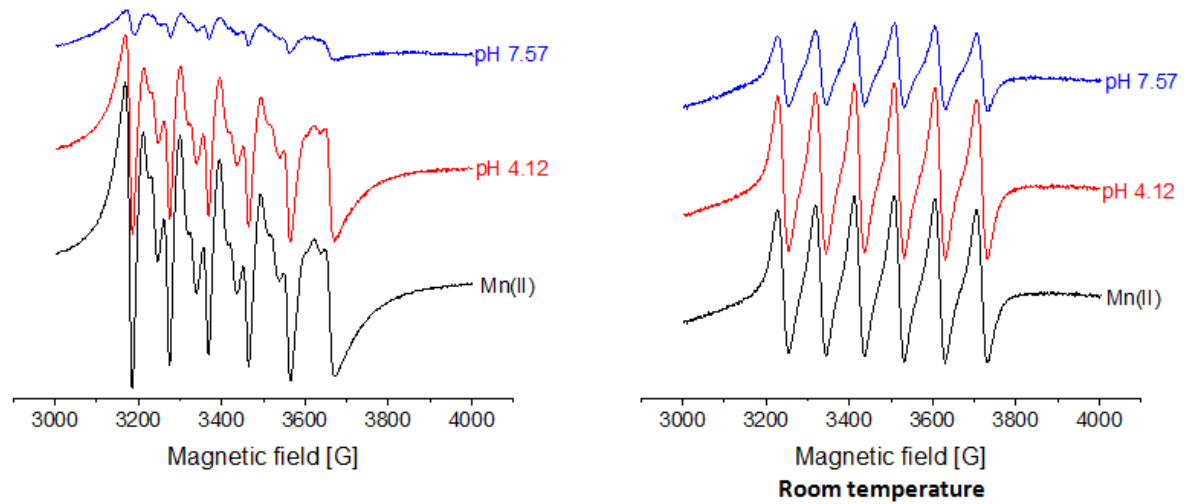

Mn(II):TMVLDEHAKG (Mid-DEH)

1.1:1
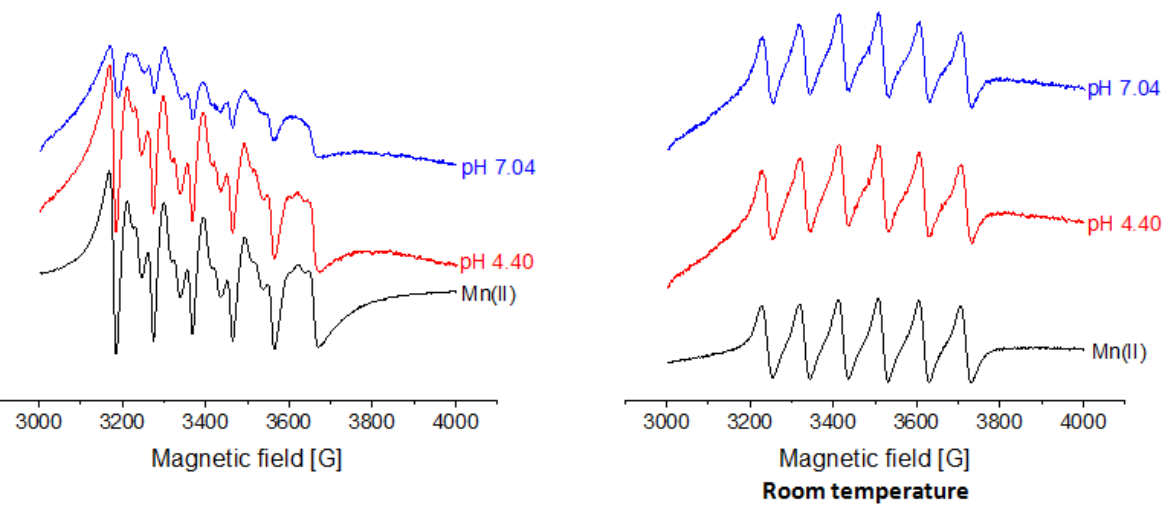

\section{Mn(II): DEHGGGGDEH (Bis-DEH)}

\section{1:1.1}
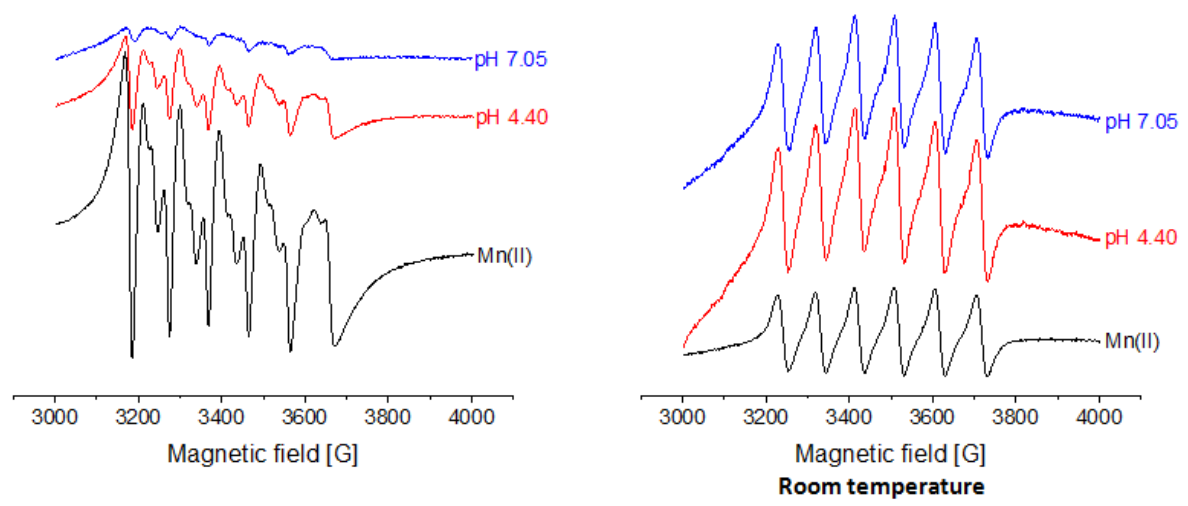

Figure S10. EPR spectra of the $\mathrm{Mn}(\mathrm{II})$-peptides system as a function of $\mathrm{pH}$. 


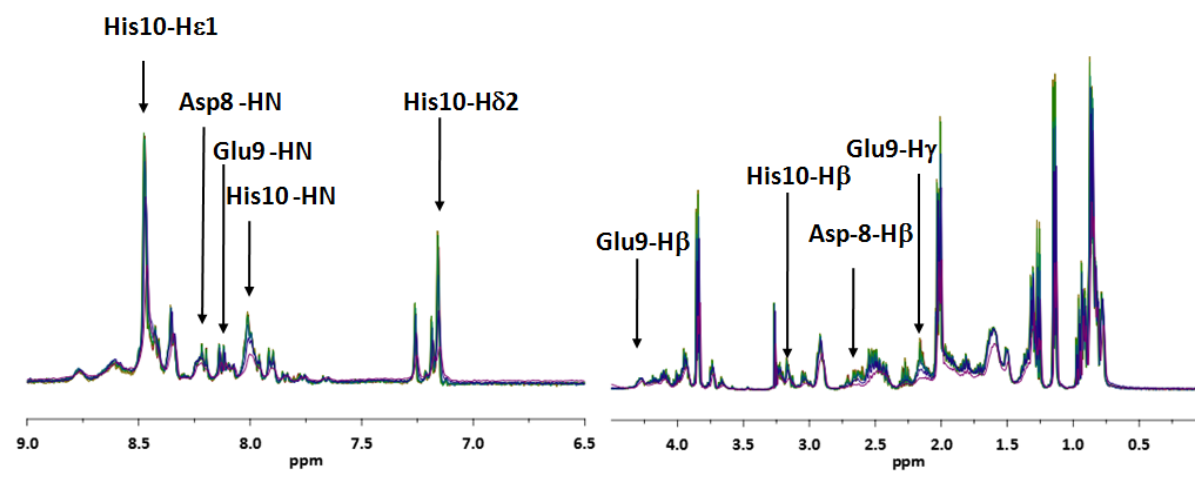

Mn(II):TMVLDEHAKG (Mid-DEH)

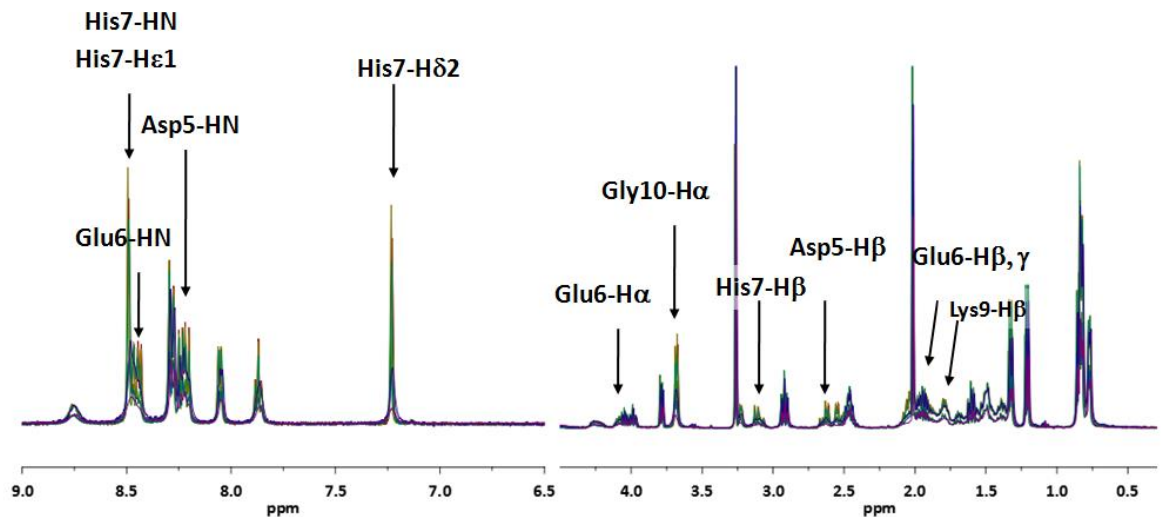

Mn(II): DEHGGGGDEH (Bis-DEH)
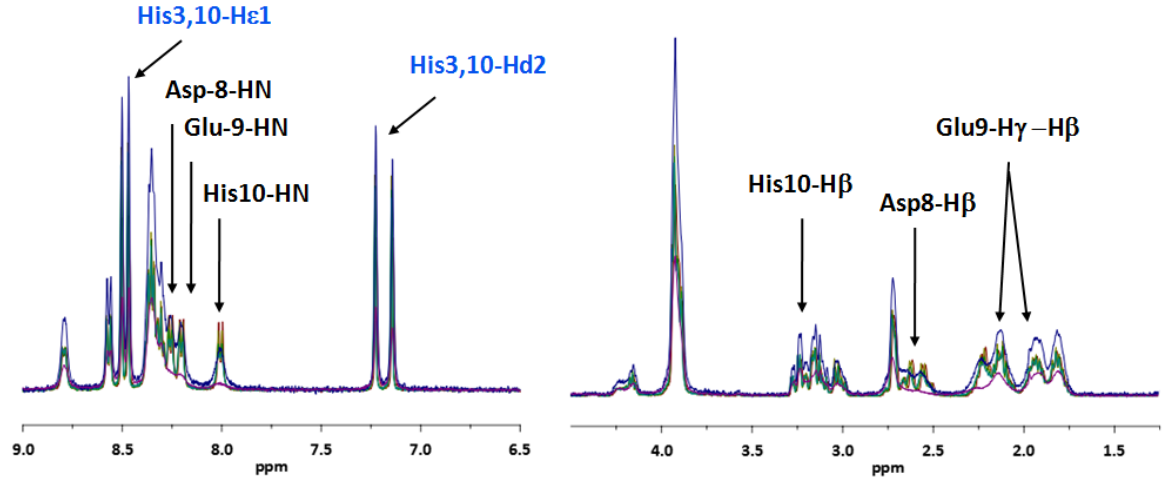

Figure S11. Superposition of 1D ${ }^{1} \mathrm{H}$ NMR spectra for GTAVMLKDEH (Term-DEH), TMVLDEHAKG (Mid-DEH) and DEHGGGGDEH (Bis-DEH) peptides by increasing substochiometric Mn(II) to ligand molar ratio, at pH 5. 

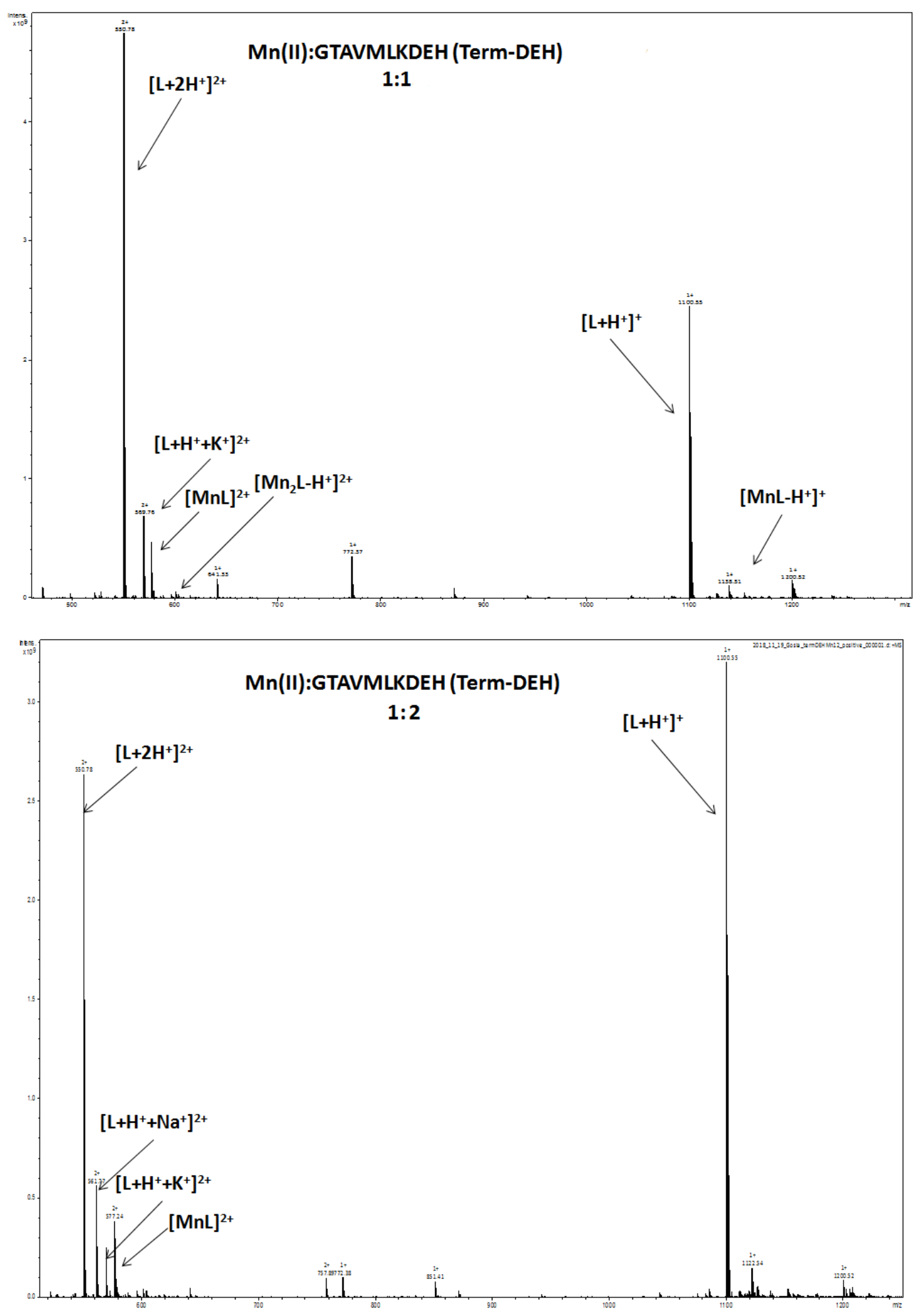

Figure S12. ESI-MS of Mn(II):GTAVMLKDEH (Term-DEH), at 1:1 and 1:2 molar ratio, and pH 7.5. 
Mn(II):TMVLDEHAKG (Mid-DEH)

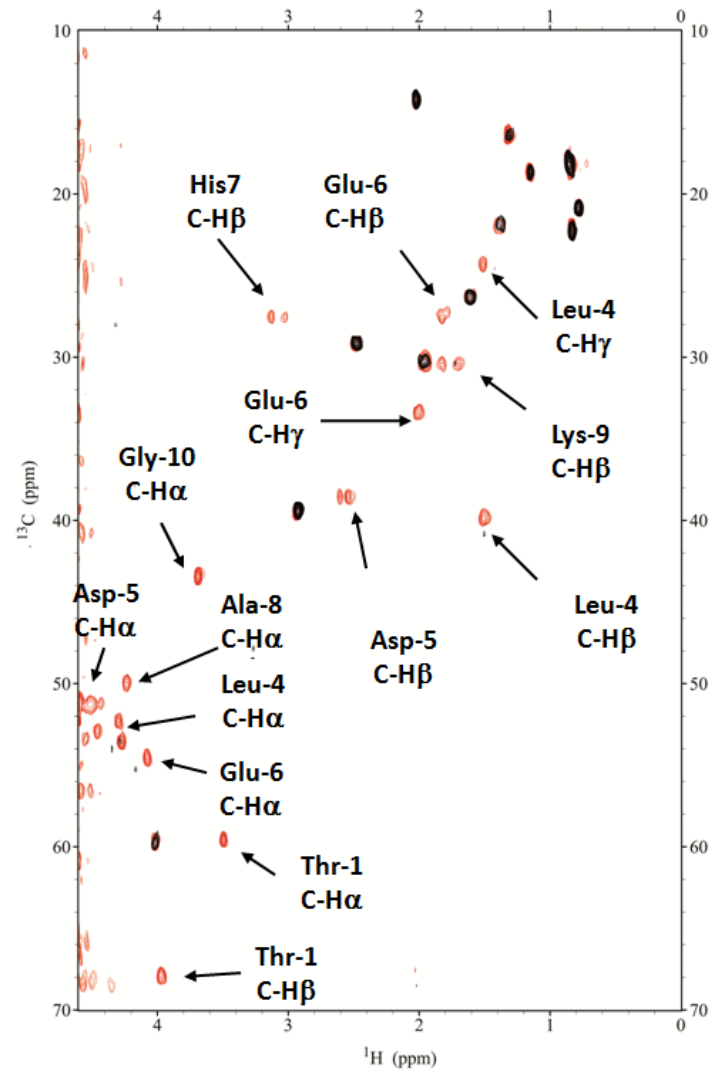

Figure S13. Aromatic and aliphatic region of ${ }^{1} \mathrm{H}-{ }^{13} \mathrm{C}$ HSQC spectrum of free Mid-DEH (red) and Mn(II): Mid-DEH at 0.1:1 molar ratio (blue) and $\mathrm{pH}$ 7.2. Disappearing peaks are labeled. 


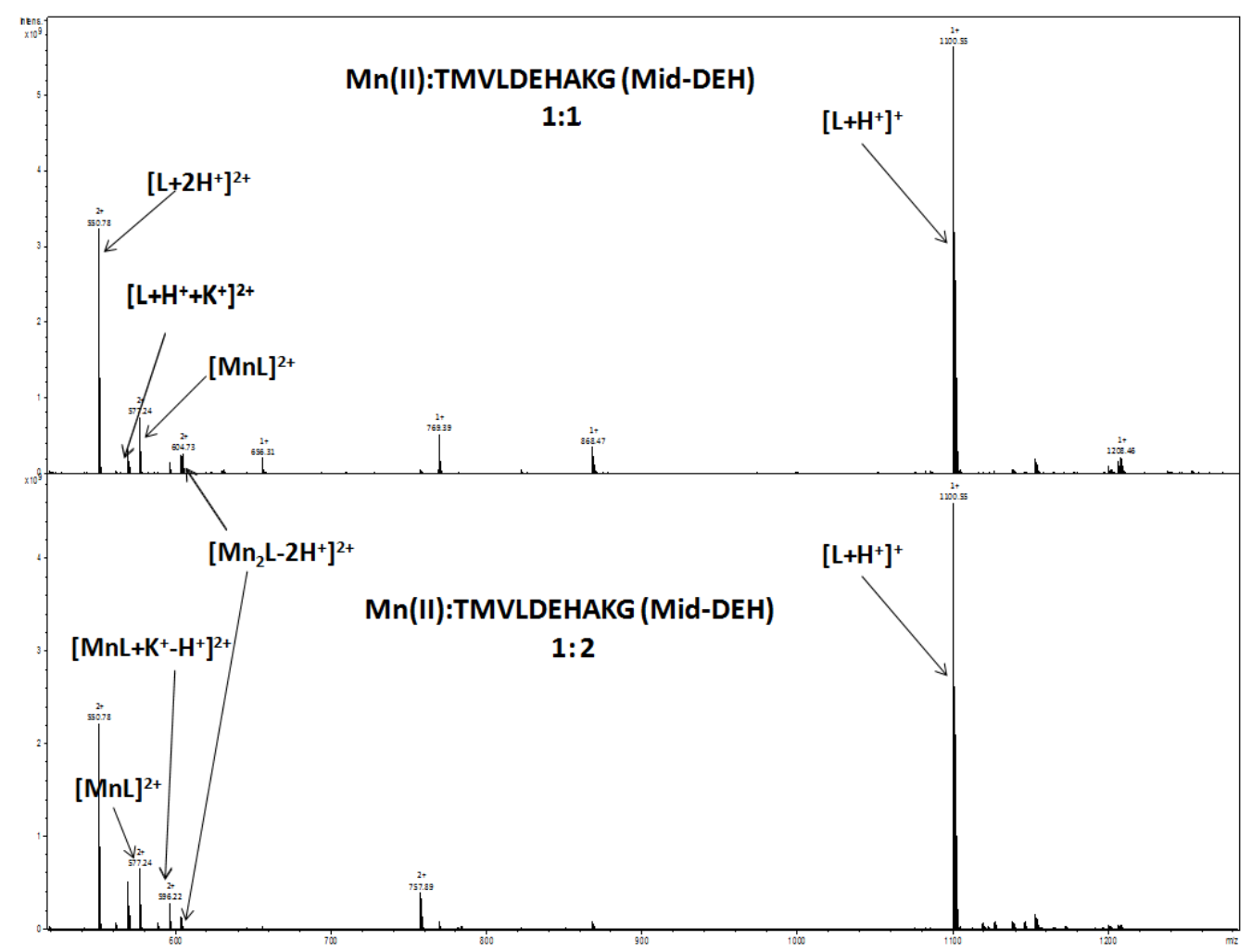

Figure S14. ESI-MS of Mn(II):TMVLDEHAKG (Mid-DEH), at 1:1 and 1:2 molar ratio, and pH 7.5. 


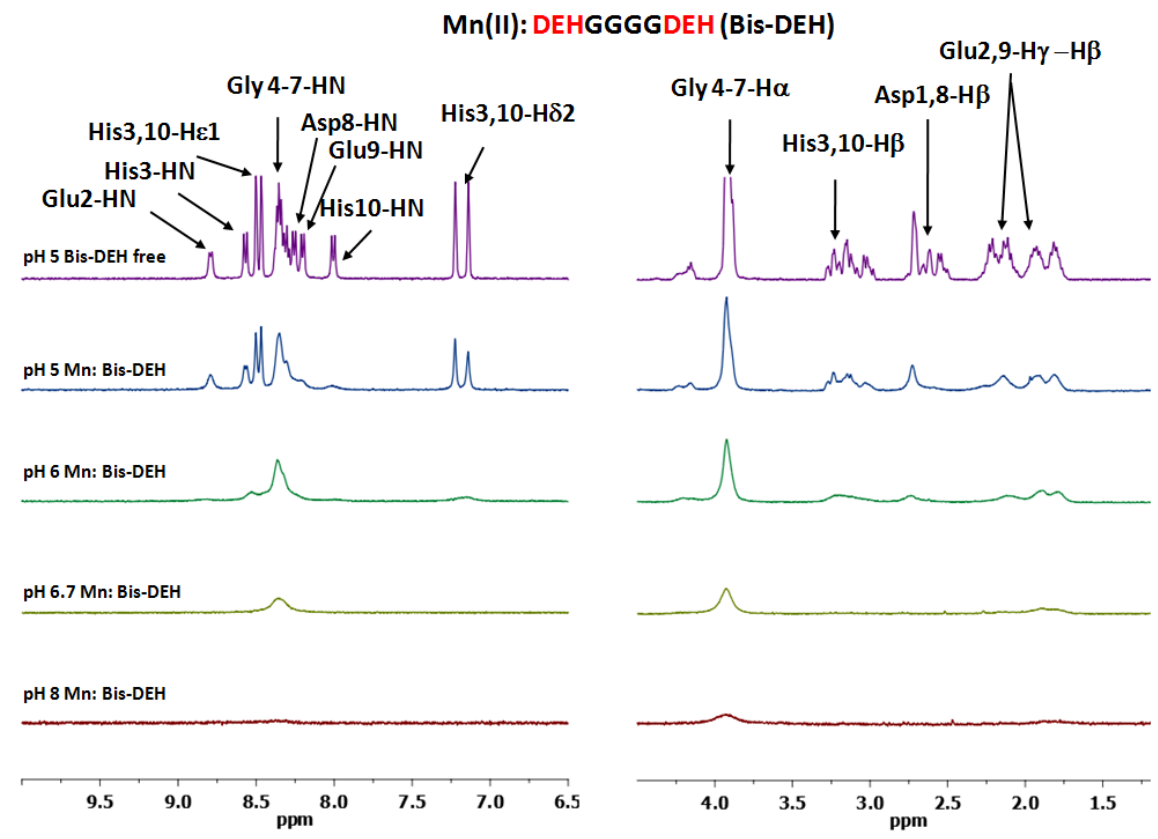

Figure S15. Comparison of $1 \mathrm{D}{ }^{1} \mathrm{H}$ NMR spectra for free DEHGGGGDEH (Bis-DEH) at pH 5, and Mn(II):Bis-DEH system (0.05:1 molar ratio) at $\mathrm{pH} \mathrm{5,6,6.7} \mathrm{and} \mathrm{8,} \mathrm{respectively.}$

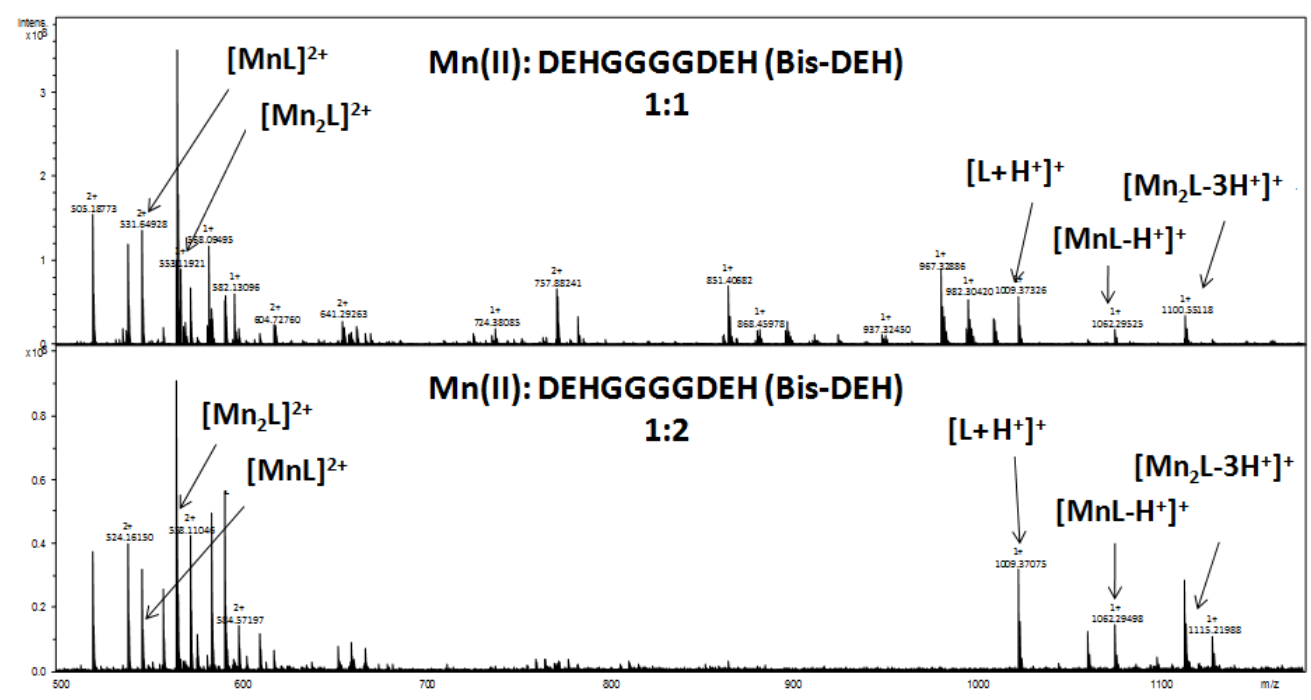

Figure S16. ESI-MS of Mn(II):DEHGGGGDEH (Bis-DEH), at 1:1 and 1:2 molar ratio, and pH 7.5. 

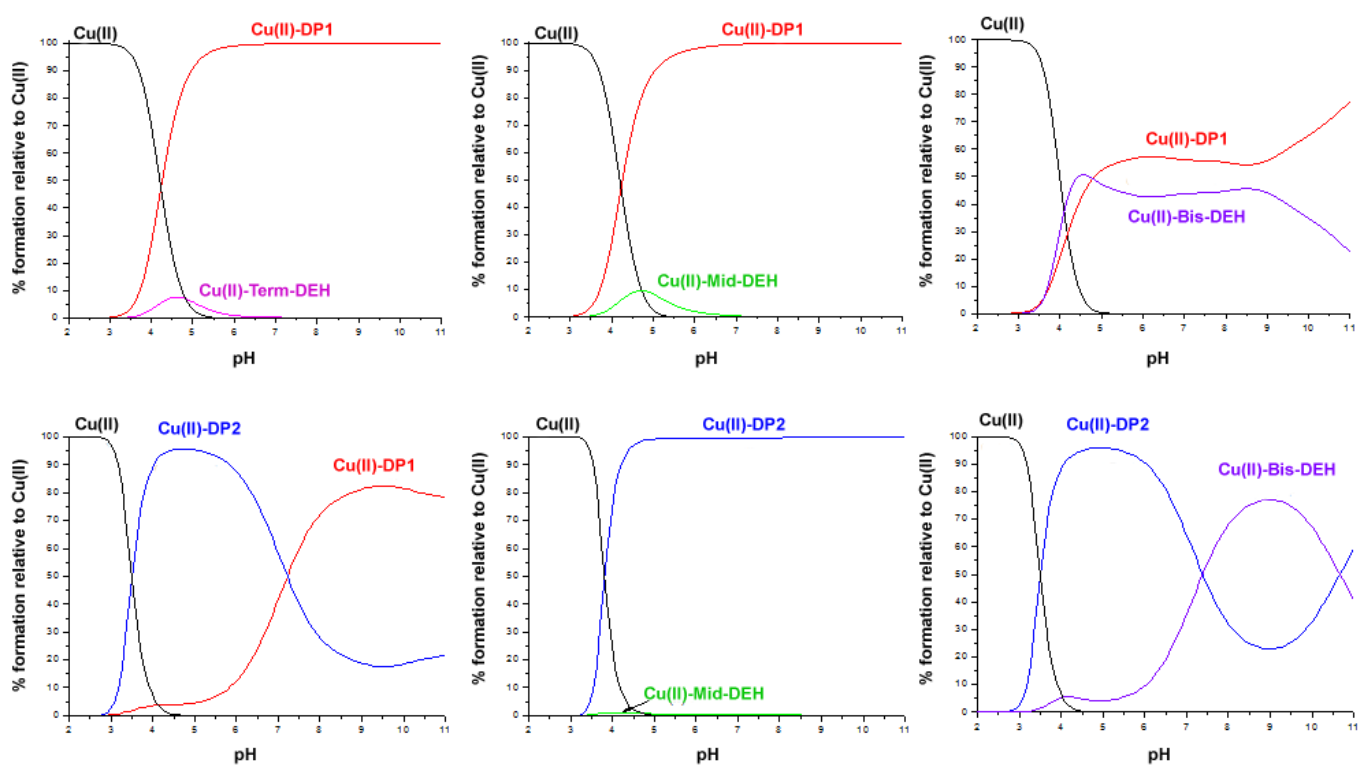

Figure S17. Competition plots for a pair of peptides between DEHGTAVMLK (DP1), THMVLAKGED (DP2), GTAVMLKDEH (Term-DEH); TMVLDEHAKG (Mid-DEH) and DEHGGGGDEH (Bis-DEH).

The calculated stability constants are applied to a theoretical situation, in which equimolar amounts of pair of peptides are competing for the binding with $\mathrm{Cu}(\mathrm{II})$ ion in 1:1:1 metal-to-ligand molar ratio. 

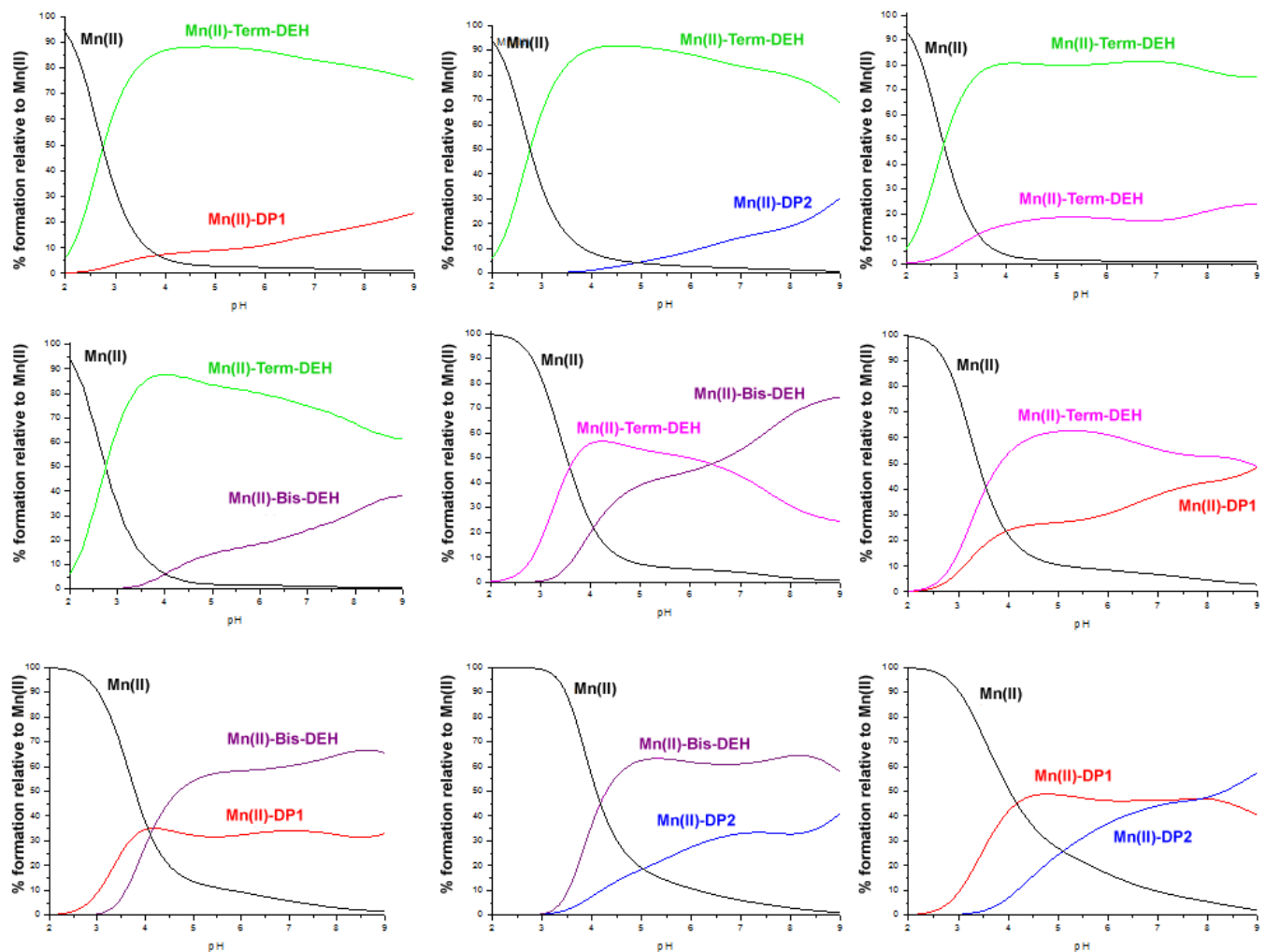

Figure S18. Competition plots for a pair of peptides between DEHGTAVMLK (DP1), THMVLAKGED (DP2), GTAVMLKDEH (Term-DEH); TMVLDEHAKG (Mid-DEH) and DEHGGGGDEH (Bis-DEH).

The calculated stability constants are applied to a theoretical situation, in which equimolar amounts of pair of peptides are competing for the binding with $\mathrm{Mn}(\mathrm{II})$ ion in 1:1:1 metal-to-ligand molar ratio. 


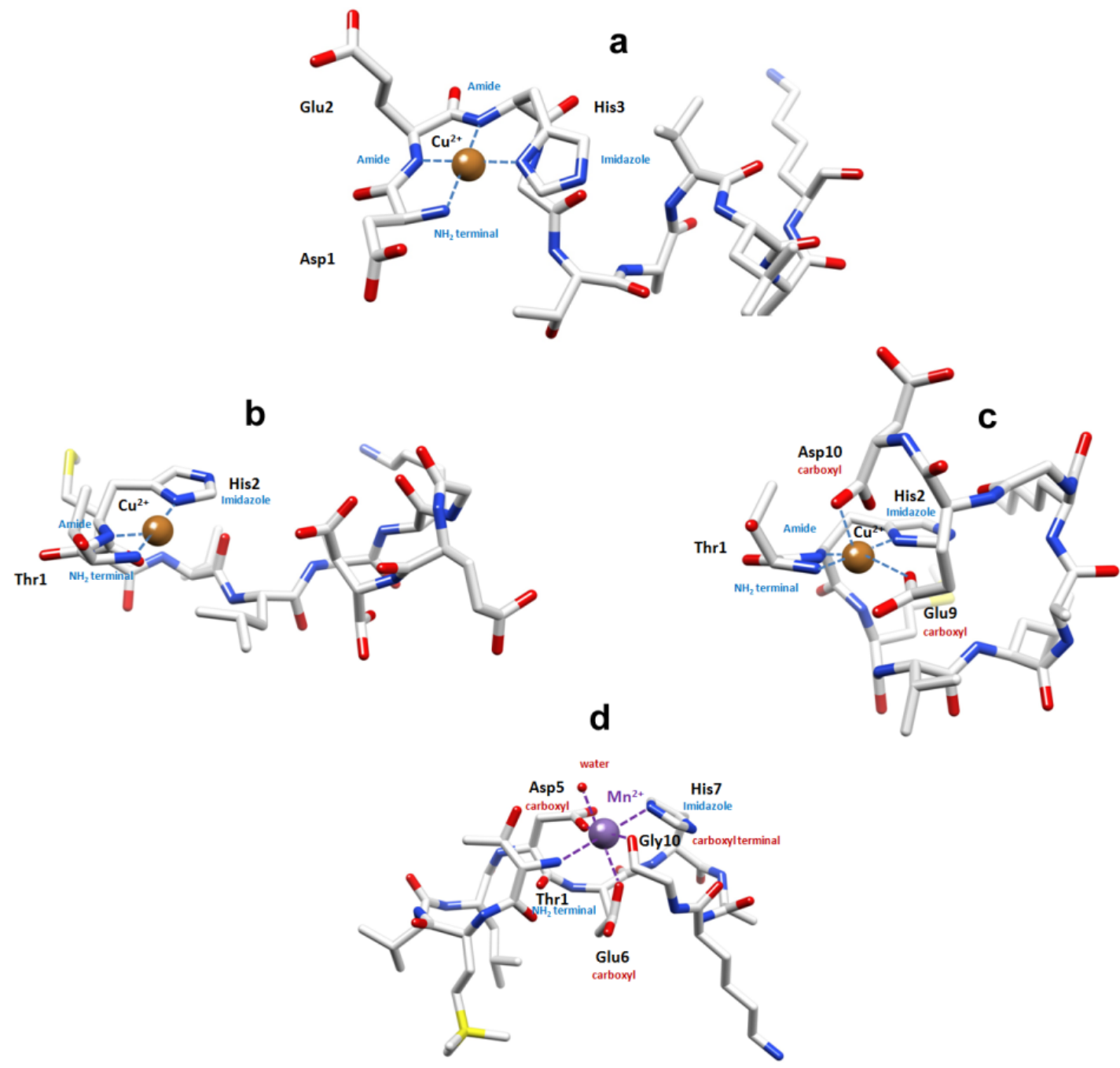

Figure S19. Structural models of a) DP1-Cu in $4 \mathrm{~N}\left\{\mathrm{~N}_{\text {term }}, \mathrm{N}_{\mathrm{im}}, 2 \mathrm{~N}^{-}\right.$amide $\}$albumin-like, b) DP2-Cu in $3 \mathrm{~N}\left\{\mathrm{~N}_{\text {term }}, \mathrm{N}_{\mathrm{im},} \mathrm{N}^{-}\right.$ amide $\}$, c) DP2-Cu in $3 \mathrm{~N} 2 \mathrm{O}\left\{\mathrm{N}_{\text {term }}, \mathrm{N}_{\mathrm{im}}, \mathrm{N}^{-}\right.$amide, $\left.2 \mathrm{O}\right\}$, and d) Mid-DEH-Mn in $2 \mathrm{~N} 4 \mathrm{O}\left\{\mathrm{N}_{\text {term }}, \mathrm{N}_{\mathrm{im}}, 4 \mathrm{O}\right\}$ coordination modes, respectively. The proposed models should be taken with appropriate caution, since they were constructed by selective imposition of a number of constraints; however, they can still represent an interesting structural hypothesis for the complexes formed in solution, in agreement with experimental evidences..

For $\mathrm{Cu}(\mathrm{II})-\mathrm{DP} 1$ model a), the $\mathrm{Cu}(\mathrm{II})$ ion was constrained in a $4 \mathrm{~N}$ square planar coordination mode using the metaldonor distances and backbone dihedral angles derived from X-ray crystal structures of aqua-((aspartic acid)-alanylhistidinyl-lysinyl)-copper(II) chloride hexahydrate (CSD Entry: OZEBOL). Moreover, meaningful ROEs involving a through-space correlation of the side-chain aliphatic protons of Glu-3 with the aromatic and aliphatic $\beta$-protons of His-3 were also used and introduced as constraints for model generation. These through-space correlations were evaluated using diamagnetic $\mathrm{Ni}(\mathrm{II})$ (at $\mathrm{pH}$ 10) to probe paramagnetic $\mathrm{Cu}$ (II) in the same square planar coordination mode. The geometry of the rest of the peptide was optimized without any constraints allowing all the atoms, bonds and dihedral angles to change simultaneously to reach the lowest overall energy within the HyperChem ${ }^{\mathrm{TM}}$ 8.0.3 program (Hypercube, Inc., Gainesville, FL, 2007). For $\mathrm{Cu}(\mathrm{II})$-DP2 (b model), the $\mathrm{Cu}(\mathrm{II})$ ion was constrained in a $3 \mathrm{~N}$ planar coordination involving $\mathrm{N}_{\text {term }}$ from Thr-1 and $\mathrm{N}_{\mathrm{im}}$ and $\mathrm{N}_{\text {amide }}^{-}$from His-2. The $\mathrm{N}-\mathrm{Cu}$ (II) distances were derived from the same template (CSD Entry: OZEBOL). Moreover, in c model, additional constraints were imposed to the carboxylic oxygen donors of Asp-9 and Glu-10 in order to be connected to the metal ion. The other residues and backbone dihedral angles have been left free to move. As for the $\mathrm{d}$ model, $\mathrm{Mn}$ (II) bound in an octahedral $\left\{\mathrm{N}_{\mathrm{term}}, \mathrm{N}_{\mathrm{im}}, 4 \mathrm{O}\right\}$ coordination mode with Thr-1 $\left(\mathrm{N}_{\text {term }}\right)$, Asp-5 $(\mathrm{O})$, Glu-6 $(\mathrm{O})$, His-7 $\left(\mathrm{N}_{\mathrm{im}}\right)$, Gly-10 $(\mathrm{O})$ and $\mathrm{H}_{2} \mathrm{O}$, the metal-donor distances were chosen using those found in the structure of MNTR mutant E11K complexed with $\mathrm{Mn}^{2+}$ in octahedral geometry (PDB 4HX4) while the position of all the other atoms and backbone dihedral angles were optimized without any constraints. 
Tables

Table S1. ${ }^{1} \mathrm{H}$ and ${ }^{13} \mathrm{C}$ resonance assignment $(\delta \mathrm{ppm})$ for DEHGTAVMLK (DP1), THMVLAKGED (DP2), GTAVMLKDEH (Term-DEH), TMVLDEHAKG (Mid-DEH) and DEHGGGGDEH (BisDEH) peptides.

\begin{tabular}{|c|c|c|c|c|c|c|c|c|c|c|}
\hline \multicolumn{9}{|c|}{ DEHGTAVMLK (DP1), pH 7.2 } \\
\hline Nucleuslresidue & D1 & E2 & H3 & G4 & T5 & A6 & V7 & M8 & L9 & K10 \\
\hline CA & 51.12 & 54.02 & 53.76 & 42.82 & 59.25 & 49.91 & 59.63 & 52.44 & 52.49 & 54.87 \\
\hline CB & 38.04 & 27.43 & 27.95 & & 67.06 & 16.47 & 30.16 & 29.27 & 39.54 & 31.16 \\
\hline CD & & & & & & & & & & 26.43 \\
\hline CD1 & & & & & & & & & 22.27 & \\
\hline CD2 & & & 117.40 & & & & & & 20.67 & \\
\hline CE & & & & & & & & 14.25 & & 39.50 \\
\hline CE1 & & & 135.60 & & & & & & & \\
\hline CG & & 33.24 & & & 18.89 & & & 30.15 & 24.16 & 21.98 \\
\hline CG1 & & & & & & & 18.31 & & & \\
\hline CG2 & & & & & & & 17.77 & & & \\
\hline HA & 4.047 & 4.206 & 4.570 & & 4.237 & 4.279 & 3.972 & 4.425 & 4.290 & 4.082 \\
\hline HB & & & & & 4.142 & & 1.954 & & & \\
\hline HB2 & 2.682 & 1.886 & 3.119 & & & & & 2.513 & & 1.729 \\
\hline HB3 & 2.592 & 1.777 & 3.013 & & & & & 2.438 & & 1.617 \\
\hline HD2 & & & 6.976 & & & & & & & \\
\hline HE1 & & & 7.815 & & & & & & & \\
\hline HG & & & & & & & & & 1.542 & \\
\hline HN & & & & 8.272 & 8.026 & 8.258 & 8.001 & 8.310 & 8.203 & 7.760 \\
\hline QA & & & & 3.921 & & & & & & \\
\hline QB & & & & & & 1.302 & & & 1.542 & \\
\hline QD & & & & & & & & & & 1.586 \\
\hline QD1 & & & & & & & & & 0.843 & \\
\hline QE & & & & & & & & 2.013 & & 2.913 \\
\hline QG1 & & & & & & & 0.840 & & & \\
\hline
\end{tabular}




\begin{tabular}{|c|c|c|c|c|c|c|c|c|c|c|}
\hline \multicolumn{9}{|c|}{ THMVLAKGED (DP2), pH 7.3 } \\
\hline Nucleus\residue & T1 & H2 & M3 & V4 & L5 & A6 & K7 & G8 & E9 & D10 \\
\hline CA & 59.51 & 53.63 & 52.68 & 59.63 & 52.05 & 49.48 & 53.66 & 42.46 & 53.45 & 52.11 \\
\hline CB & 68.00 & 28.46 & 29.09 & 30.12 & 39.77 & 16.56 & 30.48 & & 27.85 & 39.50 \\
\hline CD & & & & & & & 26.37 & & & \\
\hline CD1 & & & & & 22.14 & & & & & \\
\hline CD2 & & 116.90 & & & 20.78 & & & & & \\
\hline CE & & & 14.16 & & & & 39.36 & & & \\
\hline CE1 & & 136.00 & & & & & & & & \\
\hline CG & 18.44 & & 30.32 & & 24.20 & & 21.68 & & 33.61 & \\
\hline CG1 & & & & 18.46 & & & & & & \\
\hline CG2 & & & & 17.87 & & & & & & \\
\hline HA & 3.435 & 4.564 & 4.367 & 3.973 & 4.292 & 4.240 & 4.232 & & 4.285 & 4.287 \\
\hline HA1 & & & & & & & & 3.853 & & \\
\hline HA2 & & & & & & & & 3.970 & & \\
\hline HB & 3.952 & & & 1.931 & & & & & & \\
\hline HB2 & & 3.000 & 2.395 & & & & 1.739 & & 2.047 & 2.586 \\
\hline HB3 & & 2.996 & 2.365 & & & & 1.695 & & 1.816 & 2.477 \\
\hline HD2 & & 6.903 & & & & & & & & \\
\hline HE1 & & 7.700 & & & & & & & & \\
\hline HG & & & & & 1.517 & & & & & \\
\hline HN & & & & 8.134 & 8.263 & 8.213 & 8.234 & 8.384 & 8.208 & 7.964 \\
\hline QB & & & & & 1.514 & 1.282 & & & & \\
\hline QD & & & & & & & 1.605 & & & \\
\hline QD1 & & & & & 0.836 & & & & & \\
\hline QE & & & 2.002 & & & & 2.931 & & & \\
\hline QG & 1.111 & & 1.893 & & & & 1.382 & & 2.159 & \\
\hline
\end{tabular}




\begin{tabular}{|c|c|c|c|c|c|c|c|c|c|c|}
\hline \multicolumn{9}{|c|}{ GTAVMLKDEH (Term-DEH), pH 7.4 } \\
\hline Nucleus\residue & G1 & T2 & A3 & V4 & M5 & L6 & K7 & D8 & E9 & H10 \\
\hline CA & 41.27 & 59.21 & 49.87 & 59.66 & 52.54 & 52.40 & 53.56 & 51.75 & 53.81 & 54.62 \\
\hline CB & & 67.19 & 16.58 & 30.24 & 29.27 & 39.61 & 30.50 & 38.46 & 27.94 & 28.02 \\
\hline CD & & & & & & & 26.40 & & & \\
\hline CD1 & & & & & & 22.21 & & & & \\
\hline CD2 & & & & & & 20.74 & & & & 117.50 \\
\hline CE & & & & & 14.28 & & 39.40 & & & \\
\hline CE1 & & & & & & & & & & 134.30 \\
\hline CG & & 18.91 & & & 30.27 & 24.25 & 21.76 & & 33.58 & \\
\hline CG1 & & & & 18.23 & & & & & & \\
\hline CG2 & & & & 17.93 & & & & & & \\
\hline HA & & 4.277 & 4.293 & 3.980 & 4.428 & 4.285 & 4.234 & 4.528 & 4.198 & 4.353 \\
\hline HB & & 4.120 & & 1.951 & & & & & & \\
\hline HB2 & & & & & 2.502 & 1.527 & 1.714 & 2.617 & 1.939 & 3.129 \\
\hline HB3 & & & & & 2.439 & 1.493 & 1.674 & 2.491 & 1.777 & 2.980 \\
\hline HD2 & & & & & & & & & & 7.022 \\
\hline HE1 & & & & & & & & & & 8.120 \\
\hline HG & & & & & & 1.546 & & & & \\
\hline HN & & & 8.265 & 8.086 & 8.361 & 8.262 & 8.215 & 8.292 & 8.169 & 7.909 \\
\hline QA & 3.758 & & & & & & & & & \\
\hline QB & & & 1.305 & & & & & & & \\
\hline QD & & & & & & & 1.599 & & & \\
\hline QD1 & & & & & & 0.841 & & & & \\
\hline QE & & & & & 2.021 & & 2.916 & & & \\
\hline QG1 & & 1.148 & & & 1.939 & & 1.322 & & 2.094 & \\
\hline & & & 0.844 & & & & & & \\
\hline
\end{tabular}


TMVLDEHAKG (Mid-DEH), pH 7.2

\begin{tabular}{|c|c|c|c|c|c|c|c|c|c|c|}
\hline Nucleus\residue & T1 & M2 & V3 & L4 & D5 & E6 & H7 & A8 & K9 & G10 \\
\hline$\overline{\mathrm{CA}}$ & 59.54 & 52.94 & 59.67 & 52.32 & 51.27 & 54.51 & 53.39 & 49.93 & 53.60 & 43.45 \\
\hline$\overline{C B}$ & 67.86 & 29.08 & 30.32 & 39.87 & 38.56 & 27.33 & 27.54 & 16.42 & 30.38 & \\
\hline CD & & & & & & & & & 26.34 & \\
\hline CD1 & & & & 22.13 & & & & & & \\
\hline CD2 & & & & 20.83 & & & 117.40 & & & \\
\hline$\overline{C E}$ & & 14.22 & & & & & & & 39.48 & \\
\hline CE1 & & & & & & & 135.40 & & & \\
\hline$\overline{C G}$ & 18.66 & 30.32 & & 24.36 & & 33.41 & & & 21.97 & \\
\hline CG1 & & & 18.43 & & & & & & & \\
\hline CG2 & & & 17.99 & & & & & & & \\
\hline HA & 3.489 & 4.463 & 4.015 & 4.290 & 4.505 & 4.069 & 4.536 & 4.229 & 4.269 & \\
\hline HB & 3.962 & & 1.954 & & & & & & & \\
\hline HB2 & & & & & 2.601 & 1.837 & 3.125 & & 1.816 & \\
\hline HB3 & & & & & 2.533 & 1.791 & 3.016 & & 1.701 & \\
\hline HD2 & & & & & & & 7.010 & & & \\
\hline HE1 & & & & & & & 7.899 & & & \\
\hline HG & & & & 1.506 & & & & & & \\
\hline HN & & & 8.263 & 8.308 & 8.204 & 8.438 & 8.235 & 7.953 & 8.190 & 7.880 \\
\hline$\overline{\text { QA }}$ & & & & & & & & & & 3.690 \\
\hline$\overline{\text { QB }}$ & & 2.481 & & 1.499 & & & & 1.318 & & \\
\hline QD & & & & & & & & & 1.618 & \\
\hline QD1 & & & & 0.836 & & & & & & \\
\hline QD2 & & & & 0.787 & & & & & & \\
\hline $\mathbf{Q E}$ & & 2.027 & & & & & & & 2.930 & \\
\hline QG & 1.151 & 1.952 & & & & 2.002 & & & 1.395 & \\
\hline QG1 & & & 0.837 & & & & & & & \\
\hline
\end{tabular}




\begin{tabular}{|c|c|c|c|c|c|c|c|}
\hline \multicolumn{7}{|c|}{ DEHGGGGDEH (Bis-DEH), pH 7.0 } \\
\hline Nucleuslresidue & D1 & E2 & H3 & G4-G7 & D8 & E9 & H10 \\
\hline CA & 50.96 & 54.18 & 53.42 & 42.60 & 51.54 & 54.09 & 54.34 \\
\hline CB & 37.57 & 27.43 & 27.47 & & 38.57 & 27.44 & 27.48 \\
\hline CD2 & & & 117.50 & & & & 117.30 \\
\hline CE1 & & & 135.00 & & & & 133.70 \\
\hline CG & & 33.30 & & & & 33.43 & \\
\hline HA & 4.118 & 4.221 & 4.608 & & 4.543 & 4.156 & 4.376 \\
\hline HB2 & & 1.911 & 3.160 & & 2.602 & 1.924 & 3.159 \\
\hline HB3 & & 1.792 & 3.062 & & 2.488 & 1.798 & 3.002 \\
\hline HD2 & & & 7.069 & & & & 7.090 \\
\hline HE1 & & & 8.044 & & & & 8.332 \\
\hline HN & & & 8.419 & 8.347 & 8.255 & 8.257 & 7.955 \\
\hline QA & & & & 3.925 & & & \\
\hline QB & 2.695 & & & & & & \\
\hline QG & & 2.077 & & & & 2.119 & \\
\hline
\end{tabular}


Table S2. Spectroscopic parameters and coordination modes for the $\mathrm{Cu}(\mathrm{II})$ :peptide systems.

\begin{tabular}{|c|c|c|c|c|c|c|c|c|c|c|c|c|c|}
\hline \multirow{2}{*}{$\begin{array}{c}\text { DP1 } \\
\text { DEHGTAVMLK }\end{array}$} & \multirow[b]{2}{*}{ pH } & \multicolumn{2}{|c|}{$\mathrm{UV}-\mathbf{V i s}^{a}$} & \multicolumn{3}{|c|}{$\mathbf{C D}^{a}$} & \multicolumn{3}{|c|}{ EPR } & \multirow[b]{2}{*}{ Complex } & \multirow[b]{2}{*}{ Donors } & \multirow[b]{2}{*}{ pH } & \multirow{2}{*}{$\begin{array}{c}\text { NMR } \\
\text { Residue donors }\end{array}$} \\
\hline & & $\lambda$ & $\varepsilon$ & pH & $\lambda$ & $\Delta \varepsilon$ & pH & $\mathbf{A}_{\text {II }}$ & $\mathbf{g}_{\text {II }}$ & & & & \\
\hline $\begin{array}{c}\text { DP1 species } \\
{\left[\mathrm{CuH}_{2} \mathrm{~L}^{2+}-[\mathrm{CuHL}]^{+}\right.}\end{array}$ & & & & & & & 3.85 & 155.0 & 2.33 & & determined & & \\
\hline$[\mathrm{CuL}]$ & & & & & & & 4.95 & 208.7 & 2.18 & $4 \mathrm{~N}$ & $\left\{\mathrm{~N}_{\text {term }}, \mathrm{N}_{\text {im }}, 2 \mathrm{~N}_{\text {amide }}^{-}\right\}$ & 4.3 & $\left\{\right.$ D1 $\left.\mathrm{N}_{\text {term }}, \mathrm{E} 2 \mathrm{~N}_{\text {amide }}^{-}, \mathrm{H} 3 \mathrm{~N}_{\text {im },} \mathrm{N}_{\text {amide }}^{-}\right\}$ \\
\hline$\left[\mathrm{CuLH}_{-1}\right]^{-}$ & 7.04 & 525 & 297 & 7.04 & $\begin{array}{l}560 \\
485 \\
310 \\
272\end{array}$ & $\begin{array}{c}-0.65 \\
0.43 \\
1.20 \\
-2.80\end{array}$ & 7.20 & 208.7 & 2.18 & & $\left\{\mathrm{~N}_{\text {term }}, \mathrm{N}_{\text {im }}, 2 \mathrm{~N}_{\text {amide }}^{-}\right\}$ & $7.0-7.6$ & $\left\{\right.$ D1 $\left.\mathrm{N}_{\text {term }}, \mathrm{E} 2 \mathrm{~N}_{\text {amide }}^{-}, \mathrm{H} 3 \mathrm{~N}_{\mathrm{im},} \mathrm{N}_{\text {amide }}^{-}\right\}$ \\
\hline$\left[\mathrm{CuLH}_{-2}\right]^{2-}$ & 10.02 & 525 & 325 & 10.05 & $\begin{array}{l}560 \\
485 \\
310 \\
272\end{array}$ & $\begin{array}{c}-0.77 \\
0.43 \\
1.20 \\
-2.80\end{array}$ & 9.85 & 208.7 & 2.18 & & $\left\{\mathrm{~N}_{\text {term }}, \mathrm{N}_{\text {im }}, 2 \mathrm{~N}_{\text {amide }}^{-}\right\}$ & 9 & $\left\{\right.$ D1 $\left.\mathrm{N}_{\text {term }}, \mathrm{E} 2 \mathrm{~N}_{\text {amide }}^{-}, \mathrm{H} 3 \mathrm{~N}_{\text {im },} \mathrm{N}_{\text {amide }}^{-}\right\}$ \\
\hline$\left[\mathrm{CuLH}_{-3}\right]^{3-}$ & 10.95 & 525 & 339 & 11.07 & $\begin{array}{l}560 \\
485 \\
310 \\
272\end{array}$ & $\begin{array}{c}-0.84 \\
0.43 \\
1.20 \\
-2.80\end{array}$ & & & & $4 \mathrm{~N}$ & $\left\{\mathrm{~N}_{\text {term }}, \mathrm{N}_{\text {im }}, 2 \mathrm{~N}_{\text {amide }}^{-}\right\}$ & 10.0 & $\left\{\right.$ D1 $\left.\mathrm{N}_{\text {term }}, \mathrm{E} 2 \mathrm{~N}_{\text {amide }}^{-}, \mathrm{H} 3 \mathrm{~N}_{\mathrm{im},} \mathrm{N}_{\text {amide }}^{-}\right\}$ \\
\hline
\end{tabular}

${ }^{a}$ The reported errors on $\lambda_{\max }= \pm 2 \mathrm{~nm}, \varepsilon= \pm 5 \%$. 


\begin{tabular}{|c|c|c|c|c|c|c|c|c|c|c|c|c|c|}
\hline \multirow{2}{*}{$\begin{array}{c}\text { DP2 } \\
\text { THMVLAKGED }\end{array}$} & \multirow[b]{2}{*}{ pH } & \multicolumn{2}{|c|}{ UV-Vis } & \multicolumn{3}{|c|}{ CD } & \multicolumn{3}{|c|}{ EPR } & \multirow[b]{2}{*}{ Complex } & \multirow[b]{2}{*}{ Donors } & \multirow{2}{*}{\multicolumn{2}{|c|}{$\begin{array}{c}\text { NMR } \\
\text { Residue donors }\end{array}$}} \\
\hline & & $\lambda$ & $\varepsilon$ & pH & $\lambda$ & $\Delta \varepsilon$ & pH & $\mathbf{A}_{\text {II }}$ & $\mathbf{g}_{\text {II }}$ & & & & \\
\hline DP2 species & & & & & & & 3.13 & 119.27 & 2.414 & $\mathrm{Cu}_{(\mathrm{aq})}$ & & & DP2:Cu 1:0.02 \\
\hline$[\mathrm{CuHL}]^{+}$ & 4.58 & 597 & 169 & 4.34 & $\begin{array}{l}585 \\
300 \\
265\end{array}$ & $\begin{array}{c}0.22 \\
-0.74 \\
0.76\end{array}$ & 4.55 & 190.0 & 2.225 & & $\left\{\mathrm{~N}_{\text {term }}, \mathrm{N}_{\text {im }}, \mathrm{N}_{\text {amide }}^{-}\right\}$ & 4.25 & $\left\{\mathrm{H} 2 \mathrm{~N}_{\mathrm{im},}\left(\mathrm{E} \mathrm{O}_{\text {carb }}^{-}, \mathrm{D} 10 \mathrm{O}_{\text {carb }}^{-},\right)\right\}$ \\
\hline$[\mathrm{CuL}]$ & $7.55-8.17$ & 595 & 210 & $6.2-8.2$ & $\begin{array}{l}585 \\
300 \\
265\end{array}$ & $\begin{array}{c}0.22 \\
-0.74 \\
1.06\end{array}$ & 6.95 & 190.0 & 2.225 & & $\left\{\mathrm{~N}_{\text {term }}, \mathrm{N}_{\text {im }}, \mathrm{N}_{\text {amide }}^{-}\right\}$ & 7.0 & $\left\{\mathrm{~T} 1 \mathrm{~N}_{\text {term }}, \mathrm{H} 2 \mathrm{~N}_{\mathrm{im},} \mathrm{N}_{\text {amide, }}^{-}\left(\mathrm{E} 9 \mathrm{O}_{\text {carb }}^{-}, \mathrm{D} 10 \mathrm{O}_{\text {carb }}^{-}\right)\right\}$ \\
\hline$\left[\mathrm{CuLH}_{-1}\right]^{-}$ & $7.55-8.17$ & 595 & 210 & & t detern & & 9.08 & 190.0 & 2.225 & $3 \mathrm{~N}$ & $\left\{\mathrm{~N}_{\text {term. }} 2 \mathrm{~N}_{\text {amide }}^{-}\right\}$ & & \\
\hline$\left[\mathrm{CuLH}_{-2}\right]^{2-}$ & 10.0 & 540 & 251 & 10.1 & $\begin{array}{c}620 \\
537 \\
290.0\end{array}$ & $\begin{array}{c}-0.24 \\
0.55 \\
-1.62\end{array}$ & & & & & $\left\{\mathrm{~N}_{\text {term }}, 3 \mathrm{~N}_{\text {amide }}^{-}\right\}$ & 10.1 & $\left\{\mathrm{~T} 1 \mathrm{~N}_{\text {term }}, \mathrm{H} 2 \mathrm{~N}_{\text {amide }}^{-}, \mathrm{M} 3 \mathrm{~N}_{\text {amide }}^{-}, \mathrm{V} 4 \mathrm{~N}_{\text {amide }}^{-},\left(\mathrm{E} 9 \mathrm{O}_{\text {carb }}^{-}, \mathrm{D} 10 \mathrm{O}_{\text {carb }}^{-}\right)\right\}$ \\
\hline$\left[\mathrm{CuLH}_{-3}\right]^{2-}$ & 10.8 & 530 & 262 & 11.06 & $\begin{array}{l}620 \\
537 \\
290\end{array}$ & $\begin{array}{c}-0.24 \\
0.36 \\
-1.62\end{array}$ & & determ & & & $\left\{\mathrm{N}_{\text {term }}, 3 \mathrm{~N}_{\text {amide }}^{-}\right\}$ & & \\
\hline
\end{tabular}




\begin{tabular}{|c|c|c|c|c|c|c|c|c|c|c|c|c|c|}
\hline \multirow{2}{*}{$\begin{array}{c}\text { Term-DEH } \\
\text { GTAVMLKDEH }\end{array}$} & \multirow[b]{2}{*}{ pH } & \multicolumn{2}{|c|}{ UV-Vis } & \multicolumn{3}{|c|}{ CD } & \multicolumn{3}{|c|}{ EPR } & \multirow[b]{2}{*}{ Complex } & \multirow[b]{2}{*}{ donors } & \multicolumn{2}{|r|}{ NMR } \\
\hline & & $\lambda$ & $\varepsilon$ & pH & $\lambda$ & $\Delta \varepsilon$ & $\mathrm{pH}$ & $A_{\text {III }}$ & $\mathrm{g}_{\text {II }}$ & & & pH & Residue donors \\
\hline Term-DEH species & & & & & & & $2.80-3.09$ & 119.30 & 2.416 & $\mathrm{Cu}_{(\mathrm{ag})}$ & & & Term-DEH:Cu 1:0.02 \\
\hline$\left[\mathrm{CuH}_{2}\right]^{2+}$ & 4.96 & 715 & 25 & & deter & & 5.13 & 140 & 2.320 & $1 \mathrm{~N}$ & $\left\{\mathrm{~N}_{\mathrm{im}}\right\}$ & 5.12 & $\left\{\mathrm{D} 8 \mathrm{O}_{\mathrm{carb}}^{-}, \mathrm{H} 10 \mathrm{~N}_{\mathrm{im}}\right\}$ \\
\hline$[\mathrm{CuHL}]^{+}$ & 6.05 & 660 & 60 & 6.12 & $\begin{array}{l}695 \\
320 \\
270\end{array}$ & $\begin{array}{l}-0.07 \\
0.25 \\
-0.25\end{array}$ & 5.97 & 160 & 2.290 & $2 \mathrm{~N}$ & $\left\{\mathrm{~N}_{\mathrm{term}}, \mathrm{N}_{\mathrm{im}}\right\}$ & 6.30 & $\left\{\right.$ G1 $\left.\mathrm{N}_{\text {term }}, \mathrm{D} 8 \mathrm{O}_{\text {carb }}^{-}, \mathrm{H} 10 \mathrm{~N}_{\mathrm{im},} \mathrm{N}_{\text {amide }}^{-}\right\}$ \\
\hline$[\mathbf{C u L}]$ & 7.44 & 625 & 96 & 8.00 & $\begin{array}{l}680 \\
320 \\
270\end{array}$ & $\begin{array}{c}-0.21 \\
0.62 \\
-0.69\end{array}$ & $7.84-8.78$ & 169 & 2.230 & $3 \mathrm{~N}$ & $\left\{\mathrm{~N}_{\text {term }}, \mathrm{N}_{\mathrm{im}} \mathrm{N}_{\text {amide }}\right\}$ & 7.4 & $\left\{\right.$ G1 $\left.\mathrm{N}_{\text {term, }}, \mathrm{D} 8 \mathrm{O}_{\text {carb }}^{-}, \mathrm{H} 10 \mathrm{~N}_{\mathrm{im},}, \mathrm{N}_{\text {amide }}^{-}\right\}$ \\
\hline$\left[\mathrm{CuLH}_{-1}\right]^{-}$ & & deter & & & deter & & & etermin & & & determined & 9.0 & $\left\{\right.$ G1 $\left.\mathrm{N}_{\text {term, }}, \mathrm{D} 8 \mathrm{O}_{\text {carb }}, \mathrm{E} 9 \mathrm{~N}_{\text {amide, }}^{-}, \mathrm{H} 10 \mathrm{~N}_{\mathrm{im},} \mathrm{N}_{\text {amide }}^{-}\right\}$ \\
\hline$\left[\mathrm{CuLH}_{2}\right]^{2-}$ & 10.31 & 520 & 162 & 10.06 & $\begin{array}{l}540 \\
310 \\
275 \\
250\end{array}$ & $\begin{array}{c}-1.06 \\
1.1 \\
-2.61 \\
1.45\end{array}$ & 10.07 & 204 & 2.185 & $4 \mathrm{~N}$ & $\left\{\mathrm{~N}_{\text {temm }} / \mathrm{N}_{\mathrm{im}}, 3 \mathrm{~N}_{\text {amide }}^{-}\right\}$ & 10.1 & $\left\{\right.$ G1 $\mathrm{N}_{\text {temm, }}$ D8 $\mathrm{N}_{\text {amidec }}^{-}$E9 $\left.\mathrm{N}_{\text {amidec }}^{-} \mathrm{H} 10 \mathrm{~N}_{\text {amidd }}^{-}\right\}$ \\
\hline$\left[\mathrm{CuLH}_{-3}\right]^{3-}$ & 11.01 & 520 & 170 & 11.03 & $\begin{array}{l}537 \\
310 \\
275\end{array}$ & $\begin{array}{c}-1.11 \\
1.14 \\
-275\end{array}$ & 10.97 & 204 & 2.185 & $4 \mathrm{~N}$ & $\left\{\mathrm{~N}_{\mathrm{term}} / \mathrm{N}_{\mathrm{im}}, 3 \mathrm{~N}_{\text {amide }}^{-}\right\}$ & & \\
\hline
\end{tabular}




\begin{tabular}{|c|c|c|c|c|c|c|c|c|c|c|c|c|}
\hline \multirow{2}{*}{$\begin{array}{c}\text { Mid-DEH } \\
\text { TMVLDEHAKG }\end{array}$} & \multirow[b]{2}{*}{ pH } & \multicolumn{2}{|c|}{ UV-Vis } & \multicolumn{3}{|c|}{ CD } & \multicolumn{3}{|c|}{ EPR } & \multirow[b]{2}{*}{ Complex } & \multirow{2}{*}{\multicolumn{2}{|c|}{$\begin{array}{c}\text { NMR } \\
\text { Residue donors }\end{array}$}} \\
\hline & & $\lambda$ & $\varepsilon$ & pH & $\lambda$ & $\Delta \varepsilon$ & pH & $\mathbf{A}_{\text {II }}$ & $\mathbf{g}_{\text {II }}$ & & & \\
\hline \multirow[t]{2}{*}{ Mid-DEH species } & & & & & & & & & & & & "Mid-DEH:Cu 1:0.02 \\
\hline & & & & & & & $3.17-4.19$ & 119.3 & 2.416 & $\mathrm{Cu}_{\text {(aq) }}$ & & \\
\hline \multirow[t]{3}{*}[\mathrm{CuH}_{2}\mathbf{L}]{$^{2+}$} & 4.85 & 710 & 37 & 5.22 & 630 & -0.13 & 5.14 & 151.9 & 2.323 & $\left\{\mathrm{~N}_{\mathrm{im}}\right\}$ & 4.9 & $\left\{\mathrm{D}^{\prime} \mathrm{O}_{\text {carb }}^{-}, \mathrm{H} 7 \mathrm{~N}_{\mathrm{im},}, \mathrm{G} 10 \mathrm{O}_{\text {term-carb }}^{-}\right\}$ \\
\hline & & & & & 340 & 0.10 & & & & & & \\
\hline & & & & & 235 & -2.34 & & & & & & \\
\hline \multirow[t]{6}{*}[\mathrm{CuHL}]{$^{+}$} & 5.34 & 655 & 62 & 5.22 & 630 & -0.13 & & & & $\left\{\mathrm{~N}_{\text {term }}, \mathrm{N}_{\text {im }}\right\}$ & 5.5 & $\left\{\mathrm{D}^{\prime} \mathrm{O}_{\text {carb }}^{-}, \mathrm{H} 7 \mathrm{~N}_{\mathrm{im},}, \mathrm{G} 10 \mathrm{O}_{\text {term-carb }}^{-}\right\}$ \\
\hline & & & & & 340 & 0.10 & & & & & & \\
\hline & & & & & 235 & -2.34 & & & & & & \\
\hline & & & & 5.98 & 630 & -0.37 & & & & & & \\
\hline & & & & & 335 & 0.24 & & & & & & \\
\hline & & & & & 240 & -1.0 & & & & & & \\
\hline \multirow[t]{11}{*}[\mathrm{CuL}]{} & 7.56 & 617 & 150 & 6.47 & 610 & -0.55 & 6.24 & 170 & 2.218 & $3 \mathrm{~N} \quad\left\{\mathrm{~N}_{\text {term }}, \mathrm{N}_{\text {im }}, \mathrm{N}_{\text {amide }}^{-}\right\}$ & 7.2 & $\left\{\mathrm{~T} 1 \mathrm{~N}_{\text {term }}, \mathrm{D} 5 \mathrm{O}_{\text {carb }}^{-}, \mathrm{H} 7 \mathrm{~N}_{\mathrm{im},} \mathrm{N}_{\text {amide }}^{-}\right\}$ \\
\hline & & & & & 335 & 0.32 & & & & & & \\
\hline & & & & & 285 & -0.17 & & & & & & \\
\hline & & & & & 245 & -0.66 & & & & & & \\
\hline & & & & 7.01 & 610 & -0.56 & & & & & & \\
\hline & & & & & 335 & 0.32 & & & & & & \\
\hline & & & & & 285 & -0.19 & & & & & & \\
\hline & & & & 8.29 & 610 & -0.56 & & & & & & \\
\hline & & & & & 335 & 0.33 & & & & & & \\
\hline & & & & & 290 & -0.21 & & & & & & \\
\hline & & & & & 260 & 0.60 & & & & & & \\
\hline \multirow[t]{4}{*}{ [CuLH.1 $^{-}$} & 9.00 & 590 & 139 & 9.1 & 615 & -0.38 & 9.37 & 170 & 2.218 & $\left\{\mathrm{~N}_{\mathrm{im}}, 2 \mathrm{~N}_{\text {amide }}^{-}\right\}$ & 9.0 & $\left\{\mathrm{D} 5 \mathrm{~N}^{-}\right.$amide, $\mathrm{H} 7 \mathrm{~N}_{\mathrm{im},} \mathrm{N}^{-}$amide $\}$ \\
\hline & & & & & 325 & 0.12 & & & & & & \\
\hline & & & & & 293 & -0.18 & & & & & & \\
\hline & & & & & 258 & 1.98 & & & & & & \\
\hline \multirow[t]{5}{*}[\mathrm{CuLH}_{-2}]{$^{2-}$} & 10.48 & 520 & 170 & 10.1 & 532 & -0.64 & 10.54 & 204 & 2.172 & $\left\{\mathrm{~N}_{\mathrm{im}}, 3 \mathrm{~N}_{\text {amide }}^{-}\right\}$ & & \\
\hline & & & & & 350 & -0.23 & & & & & & \\
\hline & & & & & 313 & 0.35 & & & & & & \\
\hline & & & & & 285 & -0.60 & & & & & & \\
\hline & & & & & 260 & 2.05 & & & & & & \\
\hline \multirow[t]{5}{*}[\mathrm{CuLH}_{-3}]{$^{3-}$} & 11.00 & 520 & 178 & 11.04 & 530 & -0.91 & 11.34 & 204 & 2.172 & $\left\{\mathrm{~N}_{\mathrm{im}}, 3 \mathrm{~N}_{\text {amide }}^{-}\right\}$ & & \\
\hline & & & & & 350 & -0.19 & & & & & & \\
\hline & & & & & 312 & 0.57 & & & & & & \\
\hline & & & & & 280 & -0.90 & & & & & & \\
\hline & & & & & 258 & 1.80 & & & & & & \\
\hline
\end{tabular}




\begin{tabular}{|c|c|c|c|c|c|c|c|c|c|c|c|c|c|}
\hline \multirow{2}{*}{$\begin{array}{c}\text { Bis-DEH 1:1 } \\
\text { DEHGGGGDEH }\end{array}$} & \multirow[b]{2}{*}{ pH } & \multicolumn{2}{|c|}{ UV-Vis } & \multicolumn{3}{|c|}{ CD } & \multicolumn{3}{|c|}{ EPR } & \multirow[b]{2}{*}{ Complex } & \multirow[b]{2}{*}{ Donors } & \multirow[b]{2}{*}{ pH } & \multirow{2}{*}{$\begin{array}{c}\text { NMR } \\
\text { Residue donors }\end{array}$} \\
\hline & & $\lambda$ & $\varepsilon$ & $\mathrm{pH}$ & $\lambda$ & $\Delta \varepsilon$ & $\mathrm{pH}$ & $\mathbf{A}_{\text {II }}$ & $\mathrm{g}_{\mathrm{II}}$ & & & & \\
\hline \begin{tabular}{|l} 
Bis-DEH species \\
\end{tabular} & & & & & & & 3.18 & 119.3 & 2.416 & $\mathrm{Cu}_{(\mathrm{aq})}$ & & & \\
\hline$\left[\mathrm{CuH}_{2} \mathbf{L}\right]^{2+}$ & 4.1 & 728 & 21 & & & & 4.11 & 161 & 2.315 & $1 \mathrm{~N}$ & $\left\{\mathrm{~N}_{\mathrm{im}}\right\}$ & $3.86-4.48$ & $\mathrm{NA}$ (severe broadening) \\
\hline$[\mathrm{CuL}]$ & & detern & & & termin & & & determi & & & $t$ determined & & NA (severe broadening) \\
\hline$\left[\mathrm{CuLH}_{-1}\right]^{-}$ & $6.4-9.8$ & 521 & 172 & $6.4-9.8$ & $\begin{array}{l}560 \\
490 \\
310 \\
270\end{array}$ & $\begin{array}{r}0.66 \\
-0.39 \\
-1.05 \\
2.34\end{array}$ & $6.1-9.2$ & 207 & 2.170 & & $\left\{\mathrm{~N}_{\text {term }}, \mathbf{N}_{\mathrm{im}}, 2 \mathrm{~N}_{\text {amide }}^{-}\right\}$ & $\begin{array}{c}7.2 \\
8 \\
8.9\end{array}$ & $\begin{array}{c}\left\{\mathrm{H} 3 \mathrm{~N}_{\mathrm{im},} \mathrm{N}_{\text {amide }}^{-}, \mathrm{H} 10 \mathrm{~N}_{\mathrm{im}}\right\} \\
\left\{\mathrm{D} 1 \mathrm{~N}_{\text {term, }}, \mathrm{E} 2 \mathrm{~N}_{\text {amide }}^{-}, \mathrm{H} 3 \mathrm{~N}_{\mathrm{im},} \mathrm{N}_{\text {amide }}^{-}\right\} \\
\left\{\text {D1 } \mathrm{N}_{\text {term, }}, \mathrm{E} 2 \mathrm{~N}_{\text {amide }}, \mathrm{H} 3 \mathrm{~N}_{\mathrm{im},} \mathrm{N}_{\text {amide }}^{-}\right\}\end{array}$ \\
\hline$\left[\mathrm{CuLH}_{2}\right]^{2-}$ & $10.3-11.0$ & 521 & 172 & $10.3-11.0$ & $\begin{array}{l}560 \\
490 \\
310 \\
270\end{array}$ & $\begin{array}{r}0.66 \\
-0.39 \\
-1.05 \\
2.34\end{array}$ & 11.30 & 207 & 2.170 & $4 \mathrm{~N}$ & $\left\{\mathrm{~N}_{\text {term }}, \mathrm{N}_{\mathrm{im},} 2 \mathrm{~N}_{\text {amide }}^{-}\right\}$ & 10.5 & $\left\{\right.$ D1 $N_{\text {term, }}$, E2 $\left.N_{\text {amide }}^{-}, \mathrm{H} 3 \mathrm{~N}_{\mathrm{im},} \mathrm{N}_{\text {amide }}^{-}\right\}$ \\
\hline
\end{tabular}




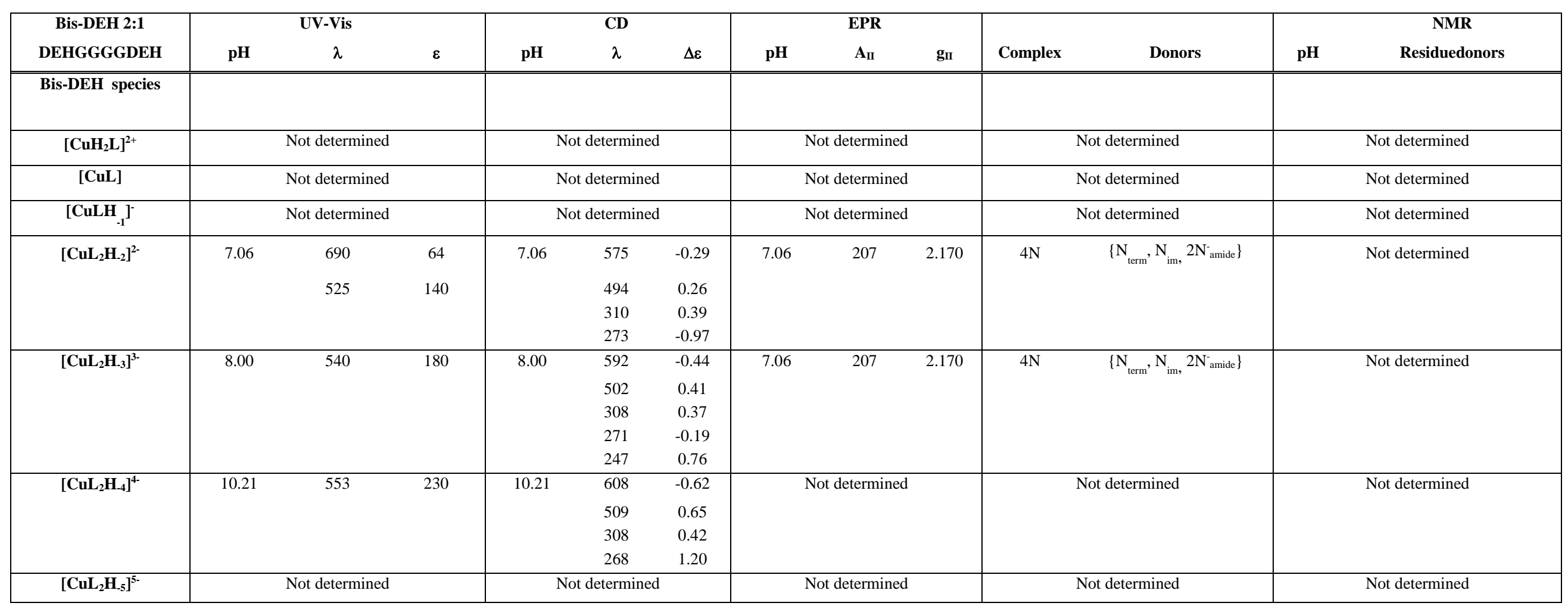


Table S3. Coordination mode for the Mn(II):peptide species.

\begin{tabular}{|c|c|c|}
\hline Peptide & Species & Residue donor \\
\hline \multirow{4}{*}{$\begin{array}{c}\text { DP1 } \\
\text { DEHGTAVMLK }\end{array}$} & {$\left[\mathrm{MnH}_{3} \mathrm{~L}\right]^{3+}$} & $\left\{\mathrm{D} 1 \mathrm{O}_{\text {carb }}^{-}, \mathrm{E} 2 \mathrm{O}_{\text {carb }}^{-}, \mathrm{K} 10 \mathrm{O}_{\text {term-carb }}^{-}\right\}$ \\
\hline & {$\left[\mathrm{MnH}_{2} \mathrm{~L}\right]^{2+}$} & $\left\{\mathrm{D} 1 \mathrm{O}_{\text {carb }}^{-}, \mathrm{E} 2 \mathrm{O}_{\text {carb }}^{-}, \mathrm{H} 3 \mathrm{~N}_{\mathrm{im}}\right\}$ \\
\hline & {$[\mathrm{MnHL}]^{+}$} & $\left\{\mathrm{D} 1 \mathrm{~N}_{\text {term }}, \mathrm{D} 1 \mathrm{O}_{\text {carb }}^{-}, \mathrm{E} 2 \mathrm{O}_{\text {carb }}^{-}, \mathrm{H} 3 \mathrm{~N}_{\mathrm{im}}\right\}$ \\
\hline & {$[\mathrm{MnL}]$} & $\left\{\mathrm{D} 1 \mathrm{~N}_{\text {term }}, \mathrm{D} 1 \mathrm{O}_{\text {carb }}^{-}, \mathrm{E} 2 \mathrm{O}_{\text {carb }}^{-}, \mathrm{H} 3 \mathrm{~N}_{\mathrm{im}}\right\}$ \\
\hline \multirow{4}{*}{$\begin{array}{c}\text { DP2 } \\
\text { THMVLAKGED }\end{array}$} & {$\left[\mathrm{MnH}_{3} \mathrm{~L}\right]^{3+}$} & $\left\{\mathrm{E} 9 \mathrm{O}_{\text {carb}}^{-}, \mathrm{D} 10 \mathrm{O}_{\text {carb }}^{-} \mathrm{O}_{\text {term-carb }}^{-}\right\}$ \\
\hline & {$\left[\mathrm{MnH}_{2} \mathrm{~L}\right]^{2+}$} & $\left\{\mathrm{H} 2 \mathrm{~N}_{\mathrm{im}}, \mathrm{E} 9 \mathrm{O}_{\text {carb }}^{-}, \mathrm{D} 10 \mathrm{O}_{\text {carb }}^{-}\right\}$ \\
\hline & {$[\mathrm{MnHL}]^{+}$} & $\left\{\mathrm{T} 1 \mathrm{~N}_{\text {term }}, \mathrm{H} 2 \mathrm{~N}_{\mathrm{im}}, \mathrm{E} 9 \mathrm{O}_{\text {carb }}^{-}, \mathrm{D} 10 \mathrm{O}_{\text {carb }}^{-} \mathrm{O}_{\text {term-carb }}^{-}\right\}$ \\
\hline & {$[\mathrm{MnL}]$} & $\left\{\mathrm{T} 1 \mathrm{~N}_{\text {term }}, \mathrm{H} 2 \mathrm{~N}_{\text {im }}, \mathrm{E} 9 \mathrm{O}_{\text {carb }}^{-}, \mathrm{D} 10 \mathrm{O}_{\text {carb }}^{-} \mathrm{O}_{\text {term-carb }}^{-}\right\}$ \\
\hline \multirow{3}{*}{$\begin{array}{c}\text { Term-DEH } \\
\text { GTAVMLKDEH }\end{array}$} & {$\left[\mathrm{MnH}_{3} \mathrm{~L}\right]^{3+}$} & $\left\{\mathrm{D} 8 \mathrm{O}_{\text {carb }}^{-}, \mathrm{E} 9 \mathrm{O}_{\text {carb }}^{-}, \mathrm{H} 10 \mathrm{O}_{\text {term-carb }}^{-}\right\}$ \\
\hline & {$\left[\mathrm{MnH}_{2} \mathrm{~L}\right]^{2+}$} & $\left\{\mathrm{D} 8 \mathrm{O}_{\text {carb }}^{-}, \mathrm{E} 9 \mathrm{O}_{\text {carb }}^{-}, \mathrm{H} 10 \mathrm{~N}_{\text {im }} \mathrm{O}_{\text {term-carb }}^{-}\right\}$ \\
\hline & {$[\mathrm{MnHL}]^{+}$} & $\left\{\mathrm{G} 1 \mathrm{~N}_{\text {term }}, \mathrm{D} 8 \mathrm{O}_{\text {carb }}^{-}\right.$E9 $\left.\mathrm{O}_{\text {carb }}^{-}, \mathrm{H} 10 \mathrm{~N}_{\text {im }} \mathrm{O}_{\text {term-carb }}^{-}\right\}$ \\
\hline \multirow{4}{*}{$\begin{array}{c}\text { Mid-DEH } \\
\text { TMVLDEHAKG }\end{array}$} & {$\left[\mathrm{MnH}_{3} \mathrm{~L}\right]^{3+}$} & $\left\{\mathrm{D} 5 \mathrm{O}_{\text {carb }}^{-}, \mathrm{E} 6 \mathrm{O}_{\text {carb }}^{-}, \mathrm{H} 7 \mathrm{~N}_{\mathrm{im}}, \mathrm{G} 10 \mathrm{O}_{\text {term-carb }}^{-}\right\}$ \\
\hline & {$\left[\mathrm{MnH}_{2} \mathrm{~L}\right]^{2+}$} & $\left\{\mathrm{D} 5 \mathrm{O}_{\text {carb }}^{-}, \mathrm{E} 6 \mathrm{O}_{\text {carb }}^{-}, \mathrm{H} 7 \mathrm{~N}_{\mathrm{im}}, \mathrm{G} 10 \mathrm{O}_{\text {term-carb }}^{-}\right\}$ \\
\hline & {$[\mathrm{MnHL}]^{+}$} & $\left\{\mathrm{T} 1 \mathrm{~N}_{\text {term }}, \mathrm{D} 5 \mathrm{O}_{\text {carb }}^{-}, \mathrm{E}^{-} \mathrm{O}_{\text {carb }}^{-}, \mathrm{H} 7 \mathrm{~N}_{\mathrm{im}}, \mathrm{G}^{-} 10 \mathrm{O}_{\text {term-carb }}^{-}\right\}$ \\
\hline & {$[\mathrm{MnL}]$} & $\left\{\mathrm{T} 1 \mathrm{~N}_{\text {term }}, \mathrm{D} 5 \mathrm{O}_{\text {carb }}^{-}, \mathrm{E} 6 \mathrm{O}_{\text {carb }}^{-}, \mathrm{H} 7 \mathrm{~N}_{\text {im }}, \mathrm{G}^{-} 0 \mathrm{O}_{\text {term-carb }}^{-}\right\}$ \\
\hline \multirow{4}{*}{$\begin{array}{c}\text { Bis-DEH } \\
\text { DEHGGGGDEH }\end{array}$} & {$\left[\mathrm{MnH}_{3} \mathrm{~L}\right]^{+}$} & $\left\{\mathrm{D} 8 \mathrm{O}_{\text {carb }}^{-}, \mathrm{E} 9 \mathrm{O}_{\text {carb }}^{-}, \mathrm{H} 10 \mathrm{O}_{\text {term-carb }}^{-}\right\}$ \\
\hline & {$\left[\mathrm{MnH}_{2} \mathrm{~L}\right]$} & $\left\{\mathrm{D} 8 \mathrm{O}_{\text {carb }}^{-}, \mathrm{E} 9 \mathrm{O}_{\text {carb }}^{-}, \mathrm{H} 10 \mathrm{~N}_{\mathrm{im}} \mathrm{O}_{\text {term-carb }}^{-}\right\}$ \\
\hline & {$[\mathrm{MnHL}]^{-}$} & $\left\{\mathrm{D} 1 / 8 \mathrm{O}_{\text {carb }}^{-}, \mathrm{E} 2 / 9 \mathrm{O}_{\mathrm{carb}}^{-}, \mathrm{H} 3 / 10 \mathrm{~N}_{\mathrm{im}}\right\}$ \\
\hline & {$[\mathrm{MnL}]^{2-}$} & $\left\{\mathrm{D} 1 \mathrm{~N}_{\text {term }}, \mathrm{D} 1 / 8 \mathrm{O}_{\text {carb }}^{-}, \mathrm{E} 2 / 9 \mathrm{O}_{\text {carb }}^{-} \mathrm{H} 3 / 10 \mathrm{~N}_{\mathrm{im}}\right\}$ \\
\hline
\end{tabular}

